\title{
7 Zusammenfassung der Ergebnisse: Analysemodell und Typologie der diskursiven Praxis des Definierens am Beispiel des Burnout-Phänomens
}

\subsection{Vorbemerkung}

In den folgenden Unterkapiteln werden die verschiedenen Analyseschritte der vorliegenden Arbeit nochmals rekapituliert und zunächst in einem Modell der diskursiven Praxis des Definierens zusammengeführt (Kap. 7.1). Am Ende dieses Analysezyklus stehen als Ergebnis dominierende Typen diskursiver Praxis des Definierens im Burnout-Diskurs, die in Kapitel 7.2 zusammenfassend als Typologie dargelegt werden. Betrachtet man diese Typen diskursiver Definierens-Praxis anschließend im Vergleich aus überindividueller, transtextueller und diachroner Perspektive, so kann man einerseits Unterschiede und andererseits Verbindungslinien zwischen ihnen erkennen. Auf dieser Basis kann man nachvollziehen, warum sich bestimmte definitorische Konzeptualisierungen über einen gewissen Zeitraum hinweg in einem Diskurs durchsetzen, obwohl es zur gleichen Zeit auch Marker des Dissenses bezüglich der Allgemeingültigkeit dieser Definitionsvarianten gibt. Das Wechselverhältnis zwischen sprachlichen Mitteln und Praktiken der >Unifizierung`, die einzelne Perspektiven auf ein Phänomen durch textübergreifende Zuordnungs- und Verknappungspraktiken stabilisieren, und sprachlichen Mitteln und Praktiken der >Agonalitätı, die diesen vermeintlichen Konsens angreifen, wird in Kap. 7.3 beleuchtet.

Anschließend wird in Kapitel 7.4 reflektiert, welchen heuristischen Mehrwert die vorgestellte Typologie und das Analysemodell für diskurslinguistische Arbeiten bieten und an welchen Stellen sich Anschlussstudien sowohl aus der Perspektive der Linguistik als auch Psychologie/Medizin anbieten. Zuletzt wird überprüft, ob die Ausgangshypothesen, die in Kapitel 1 und 4.3.4 aufgestellt wurden, sich bestätigt haben und welche gesellschaftlich-praktische Relevanz sich aus den Analyseergebnissen dieser Arbeit ergibt.

\subsection{Rekapitulation und Modellierung der Analyseschritte}

In Kapitel 4.3.4 wurde die Untersuchungsheuristik zum Definieren im Diskurs zusammengefasst und das Analysemodell einer diskursiven Praxis des Definierens prospektiv präsentiert. In diesem Kapitel werden die einzelnen Schritte, ins- 
besondere die Analyse der sprachlichen Mittel und Stimuli definitorischer Praktiken des Definierens (Kap. 6), noch einmal retrospektiv an folgendem Modell nachvollzogen:

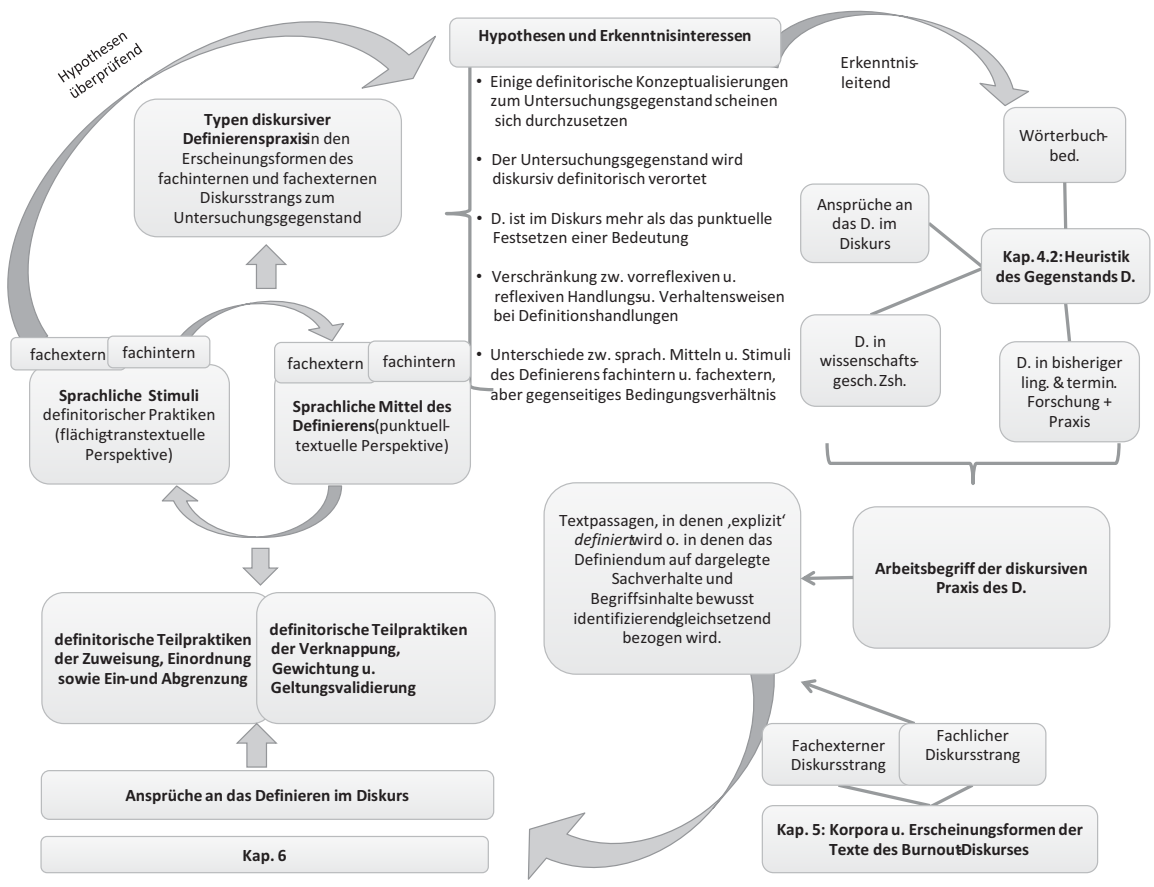

Abb. 29: Modellierung der einzelnen Schritte zur Analyse der Typen diskursiver Praxis des Definierens im Burnout-Diskurs (die Abkürzung „D.“ steht für „Definieren“).

Diesem Modell voraus gehen die Grundannahmen einer zeichengebundenen diskursiven Wissenskonstituierung (siehe Kap. 2) mit Bezug auf die Semiotik von Ch. S. Peirce und der medizinischen Semiotik (siehe Kap. 2 und 3), diskurslinguistische Ansätze im Anschluss an Foucault (Kap. 2.2.1 und 4.1.3) und die Analysekategorie diskurslinguistischer Praktik bzw. Praxis (siehe Kap. 4.1). Darauf aufbauend wurde ein induktiv-deduktives Verfahren gewählt, um sich dem Untersuchungsgegenstand des Definierens im Diskurs heuristisch zu nähern (Kap. 4.2 und in Abbildung 29 rechts oben): Literaturstudien der philosophischen, wissenschaftstheoretischen und linguistischen sowie terminologischen Literatur zum Thema „Definition“ und den dort entwickelten ,klassischen“ Definitionsformen und Definitionskriterien (siehe Kap. 4.2.3 und 4.2.4) wurden mit alltagssprachlichen Lesarten des Verbs definieren (siehe Kap. 4.2.1) und einer induktiven Analyse von 
Erwartungen und Ansprüchen an die Tätigkeit des Definierens in fachinternen und fachexternen Texten des Burnout-Diskurses verknüpft (siehe Kap. 4.2.2). Diese Schritte der Heuristik offenbarten das Forschungsdesiderat, Definieren nicht nur als reflektiert-intendierte punktuell in einem Diskurs in Erscheinung tretende Sprachhandlung (siehe Kap. 4.3.2), sondern auch als Ergebnis verschiedener, einander bedingender teil- bzw. unbewusster Verhaltensroutinen bzw. sozialer, (fach)kultureller diskursiver Praktiken, die sich punktuell und in der Fläche der Diskursstränge zeigen, zu untersuchen (siehe Kap. 4.3.3). Daran schlossen die folgenden Analysefragen an: Welche Spuren (unwillkürlicher) sozio-kultureller, diskursiver Praktiken bzw. Stimuli manifestieren sich wiederholt an verschiedenen Stellen der sprachlichen Diskursoberfläche, die auf den Prozess des Definierens einwirken? Wie wirken diese mit intendierten Definitionsbemühungen und deren sprachlichen Mitteln des Definierens in einer diskursiven Praxis des Definierens zusammen? Daraus wurden folgende Arbeitsdefinitionen diskursiver definitorischer Praktiken und Praxis entwickelt, deren Herleitung in Kapitel 4.3.4 ausführlich beschrieben wurde:

Eine weite Arbeitsdefinition (1), die intentionale und nicht bewusst intendierte Sprachverwendungsregularitäten gleich gewichtet:

Diskursive definitorische Praktiken werden als Praktiken bestimmt, die einzelne Zwecke oder Aufgaben, die aus den Funktionsansprüchen (eines Fachbereichs) an die Tätigkeit des Definierens erwachsen, mit verfügbaren (sprachlichen) Ressourcen verbinden und in ihrer Gesamtheit dadurch definitorische Wirkung entfalten, d. h. eine diskursive Praxis des Definierens bilden.

Eine engere Arbeitsdefinition (2), die aus intentionaler handlungsorientierter Perspektive Anforderungen ${ }^{747}$ an eine punktuell in einem Text erscheinende Definitionshandlung umfasst:

Der kommunikative Zweck einer Definitionshandlung besteht im strengen Sinn und insbesondere in Fachtexten darin, dass der Definierende das in Frage stehende Zeichen benennt (= Definiendum) ${ }^{748}$ und in eine Entsprechungs-, bzw. Äquivalenzbeziehung (= Definitor) zu anderen bereits bekannten Zeichen(ketten) stellt (= Definiens) $)^{749}$, wodurch er das Definiendum für sich selbst und

747 Diese Anforderungen basieren auf Kriterien, die in Kapitel 4.2.2 induktiv und in den Kapiteln 4.2.1 und 4.2.3-4.2.4 deduktiv erarbeitet wurden.

748 Nach Peirce könnte man sagen, es wird ein (neuer) Zeichenträger (sign), der im Akt der Interpretation mit einem „Zeichenobjekt“ $\mathrm{x}$ symbolisch verbunden werden soll, eingeführt; in der satzsemantischen Terminologie von P. von Polenz könnte man sagen, dass ein (neuer) „Bezugname“ genannt wird, der mit einem „Bezugobjekt“ $x$ verbunden werden soll (von Polenz ${ }^{3} 2008: 138$ ). 749 Stanaityte (2005) spricht in diesem Zusammenhang mit Bezug auf die satzsemantische Terminologie nach P. von Polenz (1985) davon, dass „das Explizieren einer Bedeutung ein Akt 
etwaige Rezipienten hinreichend genau, wesentlich bzw. in seiner Typik erfassend und mit dem Anspruch der Adäquatheit und intersubjektiver oder allgemein verbindlicher Gültigkeit feststellt oder festsetzt, von anderen Sachverhalten/Begriffen/Termini differenziert und in bestehendes Wissen einordnet.

Da die sprachlichen Mittel und Praktiken des Definierens je nach Gebrauchszusammenhang in den einzelnen untersuchten Texten des Gesamtkorpus differieren können und um etwaige Unterschiede sichtbar zu machen, wurden die Texte der Teilkorpora vor Beginn der eigentlichen Analyse daher hinsichtlich ihrer Wissensvoraussetzungen, Produzenten- und Adressatengruppen sowie ihrer damit verbundenen funktional zweckhaften Leistung, Kommunikationsbereiche und typischen Textsorten noch eingehender beurteilt (Kap. 5.3.3). Daraus ergaben sich folgende Untersuchungseinheiten: Es wurden zunächst sprachliche Mittel des Definierens in Fachlexika, Hand- und Lehrbüchern (Kap. 6.2.1.1) und danach in Fachzeitschriften und Monografien (Kap. 6.2.2.2) aus punktueller Perspektive analysiert und miteinander verglichen (Kap. 6.2.2.4). Danach erfolgte dasselbe Analyseverfahren nach sprachlichen Mitteln des Definierens im fachexternen Diskursstrang: zunächst in Pressetexten und populärwissenschaftlichen Zeitschriften (Kap. 6.2.3.1) und anschließend in öffentlichen enzyklopädischen und medizinischen Onlineportalen (Kap. 6.2.3.2).

Die größten Unterschiede zeigten sich zwischen sprachlichen Mitteln des Definierens in den Fachlexika gegenüber sprachlichen Mitteln des Definierens in medialen Magazinberichten. Auf die verschiedenen Typen diskursivdefinitorischer Diskurspraxis wird im folgenden Kapitel eingegangen. Der Einstieg in die Analyse erfolgte an Textstellen der jeweiligen Teilkorpora, in denen der Begriff `Burnout von den Autorinnen und Autoren der Texte entweder explizit als Definitionsgegenstand behandelt wurde (z. B. dadurch dass der Abschnitt mit Definition überschrieben ist) oder in denen der Ausdruck Burnout bewusst in eine Entsprechungs-, bzw. Äquivalenzbeziehung zu anderen dargelegten Sachverhalten und Begriffsinhalten gestellt und damit diesen zugewiesen wurde. Der erste Analyseeinstieg erfolgte demnach aus sprachhandlungsorientierter Per-

des doppelten Referierens ist: zunächst wird ein Bezug zu einer Referenzstelle hergestellt, worauf dann der zweite Akt des Referierens folgt, indem auf die gleiche Referenzstelle ein weiterer Bezug genommen wird, der sich von dem ersten dadurch unterscheidet, dass er andere Referenzausdrücke zur gleichen Bezugsstelle verwendet“ (Stanaityte 2005: 97). Beim Definieren werden zwei Zeichen direkt aufeinander bezogen. Da Zeichen, wie Bär (2015: 6) mit Bezug auf die Etymologie des Ausdrucks Zeichen ausführt, „,nichts anderes als zeigend bzw. zeichenhaft handeln“ heißt, könnte man beim Akt des Definierens davon sprechen, dass das Definiendum-Zeichen und das Definiens-Zeichen in dieselbe Richtung zeigen (vgl. ebd.). 
spektive (vgl. Kap. 4.3.2). In den Zusammenfassungskapiteln (6.2.2.4 und 6.2.3.3) wurden die sprachlichen Mittel des Definierens im fachinternen und fachexternen Diskursstrang einander gegenübergestellt und ihre Wirkzwecke wurden auf die induktiv erschlossenen abstrakt-idealen Ansprüche an die Tätigkeit des Definierens (Kap. 4.2.2) bezogen. Daraus ergaben sich erste Teilpraktiken mittlerer Abstraktion, die einzelne Zwecke oder Aufgaben, die aus den DefinitionsAnsprüchen (d.h., was Definitionen leisten und bewirken sollen) erwachsen, mit verfügbaren sprachlichen Ressourcen verbinden und die sich zu zwei Überkategorien definitorischer Teilpraktiken gruppieren ließen: 1.) definitorische Praktiken der Zuweisung, Einordnung sowie Ein- und Abgrenzung; 2.) definitorische Praktiken der Verknappung, Gewichtung und Geltungsvalidierung. Die genauen Analyseschritte können in den Kapiteln 6.2.2.4 und 6.2.3.3 nachvollzogen werden. An diesen ,punktuellen' Analysezugang schloss sich in den Folgekapiteln die Analyse definitorischer Praktiken aus praxeologisch-phänomenorientierter Perspektive in der Fläche der Diskursstränge an (vgl. Kap. 4.3.3 und Kap. 6.2.4). In diesen Kapiteln konnte gezeigt werden, dass unwillkürliche fachkulturelle und soziale Praktiken, die sich an der Diskursoberfläche durch Spuren manifestieren, ebenfalls definitorische (Teil)-Zwecke (wie z.B. etwas zu klassifizieren, einzugrenzen, wesentliche Merkmale $\mathrm{zu}$ transportieren oder eine allgemein gültige Definition zu bestätigen) stimulieren können und dadurch die explizitintentionalen Definitionsakte verstetigen und verstärken können.

In folgendem Unterkapitel werden nun zunächst die dominierenden Typen diskursiver Definierenspraxis bestimmt (siehe oben links im Analysemodell, Abb. 29), die sich aus der Durchführung dieses Analysemodells im Burnoutdiskurs fachintern und fachextern ergeben haben.

\subsection{Dominierende Typen diskursiver Praxis des Definierens im Burnout-Diskurs - Ein 11-Punkte-Modell}

Am Ende des vorgelegten Analyseprogramms steht die Frage, ob sich aus den Analyseergebnissen Typen diskursiver Definierens-Praxis in den Erscheinungsformen des fachinternen und fachexternen Diskursstrangs herauskristallisieren lassen. Diese Einteilung orientiert sich an den folgenden aufeinander aufbauenden Fragen:

1) In welchem Kontext erscheint die Definition: Unter welchen öffentlichmedialen oder fachlichen Kommunikationsbedingungen und damit verbundenen medialen und fachkulturellen Praktiken wird definiert? (vgl. Kap. 5.3 dieser Arbeit: In welchem Publikationsmedium, mit welchen Produzentenund anvisierten Adressatengruppen (z. B. Fachleute an (angehende) Fach- 
leute oder Fachleute/Wissensvermittler/Journalisten und Journalistinnen an fachexterne Öffentlichkeit), in welchen Textsorten mit welcher dominanten Textfunktion (z. B. Lexikonartikel, Übersichtsartikel in einer Fachzeitschrift, Problemdarstellung in Tageszeitung, etc.), unter welchen fachlichen und gesellschaftlichen Wissensvoraussetzungen?)

2) Durch welche sprachlichen Mittel (von der Textabschnitts- über die Satz-, (Mehr-)Wort bis zur Morphemebene) wird punktuell definiert? Ergeben sich aus diesen sprachlichen Mitteln des Definierens unterschiedliche Definitionsentfaltungstypen ${ }^{750}$ ? (siehe dazu Kap. 6.2.2 und 6.2.3 und die Zusammenfassungskap. 6.2.2.4 u. 6.2.3.3)

3) Welche übergeordnete Interpretationsperspektive bzw. welches Metakonzept (`Zustand`, >Vorgang`, >Ereignis`) und welche Grundkonzepte des jewei-

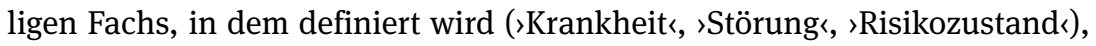
werden durch die definierende(n) Proposition(en) (= Definiens) und die Definitionsentfaltungen konstatiert und in welcher Weise mit aufgerufen?

4) Welche definitorischen Teilpraktiken mittlerer Abstraktion sind in der jeweiligen Definitionsentfaltung (besonders) präsent (z. B. |doppelt referieren/ zuweisen|, |kategorisieren|, |klassifizieren|, |Merkmale charakterisieren|, |ein typisches Beispiel erzählen|, leine Klassifizierung zurückweisen und ersetzen|, |ein Beispiel referieren/erzählen|, |stabile Abgrenzung von Vergleichswerten stimulieren|, |verallgemeinern|, |Merkmale gewichten/hervorheben|, |generalisieren|, |als faktisch-sichere Aussage qualifizieren|, |als in geltenden Taxonomien anerkannte Aussage qualifizieren|)? (Vgl. zur Notation und Bezeichnung der Praktiken die Tabellen 2.1; 2.2 in Kap. 6.2.2.4 und die Tabellen 5.1 und 5.2 in Kap. 6.2.3.3)

5) Kann man auf dieser Basis den Definitionen illokutionäre Grundtypen bzw. Grundfunktionen zuordnen?

6) Welche Ansprüche an die Tätigkeit des Definierens bzw. Definitionszwecke werden durch die jeweilige Definition/Definitionsentfaltung in besonderem Maße/weniger erfüllt? (vgl. Kap. 4.2.2 und Kap. 6.2.2.4 und 6.2.3.3)

7) Wird das Definiens durch die jeweilige definitorische Proposition (punktuelle Perspektive) und/oder durch Praktiken der Zuweisung, Einordnung, sowie Ein- und Abgrenzung im transtextuellen Diskursgeflecht (flächige Perspektive) eindeutig einem fachlichen oder gesellschaftlichen Wissensbereich zugewiesen?

750 Diese orientieren sich an linguistischer Literatur zu Grundformen der thematischen Entfaltung und Vertextungsmustern nach Konerding (2005: 9ff.), Brinker/Cölfen/Pappert ( ${ }^{8} 2014$ : 60ff.), Müller (2007: 77ff.), Gansel/Jürgens ( ${ }^{3} 2009:$ 148ff.) mit Bezug auf Heinemann/Viehweger (1991: 237ff.) und Sandig (1986: 184f.). 
8) Werden die sozial- und fachkulturellen Praktiken und Normvorstellungen, die in einzelnen Definitionen inferenziell als implizite Wissensbestände zur Abgrenzung von sachverwandten Konzepten/Sachverhalten verwendet werden, über verschiedene Texte des fachlichen und fachexternen Diskursstrangs hinweg in gleicher oder unterschiedlicher Weise evoziert?

9) Wird die Definition im Text als einzige Definition oder neben anderen Definitionen präsentiert? Wird sie gleichberechtigt oder von den anderen Definitionen in ihrer Geltung hervorgehoben präsentiert?

10) Weist die Definition Praktiken der |Gewichtung| von Definitionsattributen oder andere sprachliche Merkmale der |Zusammenfassung| oder |Verdichtung| auf? (z. B. durch typografische Hervorhebung, Ausdrücke wie Kernmerkmale etc.)

11) Wird auf die Definition intertextuell Bezug genommen und wenn ja von welchen Diskursakteuren und in welchen textuellen Erscheinungsformen? Welche Durchsetzungskraft bzw. Wirkmächtigkeit zeigt eine Definition im Horizont des Gesamtdiskurses?

Die Kriterien 1-6 basieren auf den Analysen der sich punktuell in einzelnen Textpassagen zeigenden sprachlichen Mittel und Praktiken mit definitorischer Funktion. Die Kriterien ab Frage 7 ergänzen diese beruhend auf den Analysen sprachlicher Stimuli definitorischer Praktiken im weiteren transtextuellen Geflecht im fachinternen und fachexternen Diskursstrang. Diese letzten Kriterien (7-11) bedingen die Durchsetzungskraft bzw. Wirkmächtigkeit einer Definition in einem Diskurs. Sie sind potenzielle Wirkmechanismen der Unifizierung, die dazu führt, dass divergierende Konzepte im Diskurs weniger sichtbar werden bzw. einige Definitionsvarianten sich transtextuell durchzusetzen scheinen und dadurch der Eindruck von Übereinstimmung entsteht (vgl. zum Konzept der Unifizierung Kap. 6.2.1 und 6.2.4.2 dieser Arbeit). In folgendem Kapitel 7.3 werden die Analysekategorien der >Unifizierung` und >Agonalität` (Felder 2015; Mattfeldt 2018) miteinander verglichen und im Rahmen der erfolgten Untersuchung aufeinander bezogen.

Das diskurslinguistisch-typologische Verfahren ${ }^{751}$ zur Bildung von Untertypen der diskursiven Praxis des Definierens, also die Anwendung der Fragen 1-11 auf die untersuchten Korpusbelege, beruht auf den Einzel-Analysen des Großkapitels 6. Die dort analysierten sprachlichen Mittel, Stimuli und Praktiken des Definierens werden miteinander verglichen, um Ähnlichkeiten und Unterschiede in ihrer Kombination je nach öffentlich-medialen oder fachlichen Kommunikati-

751 Zum Typologiebegriff dieser Arbeit siehe Kapitel 1.3 dieser Arbeit. 
onsbedingungen (und den damit einhergehenden Zielgruppen und Textsorten), Ko- und Kontext und Stellung im diskursiven „Textnetz“ (vgl. Felder 2012: 122) zu beschreiben. Es werden im Folgenden die dominanten Typen diskursiver Praxis des Definierens im Burnout-Diskurs in kompakter Form dargestellt. Sie charakterisieren sowohl Form, Funktion und Inhalt der jeweiligen punktuell erscheinenden Definition (Fragen 1-6) als auch deren transtextuelle Wirkkraft im Diskurs (Fragen 7-11). Die komprimiert beschriebenen eingerückt präsentierten Typen werden in den Textabschnitten danach jeweils erläutert. Die |Notation| mit senkrechten Strichen markiert Praktiken mit definitorischer Funktion, siehe dazu auch Kap. 6.2.2.4 und 6.2.3.3. Unterschiede, die sich zwischen sehr ähnlichen Untertypen ergeben, werden kursiv abgesetzt.

\section{TYP 1a: Eine sich deskriptiv entfaltende singulär erscheinende >Zustands $\triangleleft / /$ Gat- tungs<-Definition,}

- $\quad$ die |den zu definierenden Ausdruck/Begriff/Sachverhalt einem nominalen Definiensausdruck höherer Abstraktion/einer Überkategorie zuweist $\left.\right|^{752}$ und dadurch |sortal kategorisiert| ${ }^{753}$,

752 Zumeist über Formulierungen wie mit $x$ wird $y$ bezeichnet, $x$ ist $y$; unter $x$ versteht man $y$; $x$ beschreibt $y$; .... Siehe dazu Kap. 6.2.2.1, 6.2.2.2 und die Tab. 1 im Kapitel 6.2.2.4. Es wird an dieser Stelle bewusst nicht der Terminus genus proximum verwendet, da mit diesem Terminus in philosophischer und terminologischer Literatur Zusatzannahmen von klar abgrenzbaren festumrissenen Begriffsmerkmalen und (onto)logischen Begriffsbeziehungen verbunden sind, die in der formulierten Strenge auch in den untersuchten Fachlexika, Hand- und Lehrbüchern des Burnout-Diskurses selten eingehalten werden. Zur Kritik neuerer terminologischer Ansätze und empirischer Arbeiten zum Gebrauch von Fachwörtern bzw. Termini (vgl. Sager 1990, Temmerman 2000; Müller/Behr/Steffek 2019) an diesem klassischen Definitionsschema siehe ausführlich Kapitel 4.2.4 dieser Arbeit.

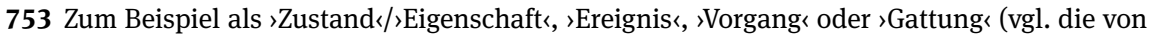
Konerding (2005: 16) beschriebenen „Prädikatorenschemata“ und die verschiedenen „Prädikatsklassen“ bei von Polenz (32008: 159-167). Das Konzept `Handlung` wird in Burnout-Definitionen als dominantes Metakonzept kaum realisiert, obwohl es in der transitiven und reflexiven Lesart des Verbs (sich) ausbrennen angelegt ist (vgl. Kap. 6.1.2). Im Rahmen der Analyse semantischer Rollen (vgl. Kap. 6.2.4.2) wurde gezeigt, dass es im Rahmen der Burnout-Definitionen wenig 'Handlungen ‘ von Personen gibt, denen die semantischen Merkmalen der 'Absicht', 'Kontrolle' und Bewusstheit' vollumfänglich zukommen (vgl. Primus 2012: 18ff.) oder die nicht negiert oder eingeschränkt werden. In manchen Belegen wird den Personen aber eine Teilverantwortlichkeit und damit Handlungsmacht bei der Entstehung von `Burnout` zugeschrieben. Diese Textstellen beziehen sich dann meist auf eine sübermäßige Arbeitstätigkeit zu Beginn`, z. B. in der ZEIT vom 20.09.2007 (= Werdes 2007, im QV unter 8.1.6): „Zu Beginn ist Burnout aber zum Teil auch hausgemacht: weil Menschen zu viel von sich verlangen oder sich zu sehr in die Arbeit stürzen.“ Zudem werden die Personen für die Verhinderung und Überwindung von `Burnout` insbesondere in fachexternen Ratgebertexten als handelnde Personen angesprochen, wie in folgendem 
- die |Hauptmerkmale charakterisiert|,

- $\quad$ die teilweise lauf typische Personengruppen generisch referiert|, |charakteristische Zusammenhänge des Auftretens beschreibt|, |Ursache-Wirkungszusammenhänge expliziert|,

- $\quad$ die lin einem Fachlexikon als einzige Definition präsentiert| und |intertextuell häufig/ selten zitiert/reformuliert| wird,

- die sich |(implizit) auf durchschnittliche menschliche und gruppenspezifische Vergleichswerte und gesellschaftliche und kulturelle Erwartungsnormen bezieht|, die sich auch in anderen Texten des Diskurses in ähnlicher Form wiederfinden und dadurch eine |stabile Ein- und Abgrenzung des zu definierenden Begriffs/Sachverhalts von diesen Vergleichswerten stimulieren|,

- $\quad$ mit repräsentativer Grundfunktion. ${ }^{754}$

\section{TYP 1b: Eine sich deskriptiv entfaltende hervorgehoben erscheinende `Zustands $\triangleleft-D e-$ finition,}

- $\quad$ die /den zu definierenden Ausdruck/Begriff/Sachverhalt einem nominalen Definiensausdruck höherer Abstraktion/einer Überkategorie zuweist| und dadurch |sortal kategorisiert|,

- die |Hauptmerkmale charakterisiert|,

- die teilweise: |auf typische Personengruppen generisch referiert|, |charakteristische Zusammenhänge des Auftretens beschreibt|, |Ursache-Wirkungszusam-menhänge expliziert|, |die Hauptmerkmale mit anderen Konzepten vergleicht|,

- $\quad$ die lin einem Hand- oder Lehrbuch, Fach-Übersichtsartikel oder einer fachlichen Originalarbeit (als hervorgehobene) Definition singulär oder neben anderen Definitionen präsentiert| und |intertextuell (häufig/selten) zitiert/reformuliert| wird // Typ 1c: die lin einem enzyklopädischen Online-Portal zu Beginn des Onlinebeitrags präsentiert wird| und meist |auf eine Definition des Typs 1 a intertextuell Bezug nimmt|,

- die sich |(implizit) auf durchschnittliche menschliche und gruppenspezifische Vergleichswerte und gesellschaftliche und kulturelle Erwartungsnormen bezieht|, die sich häufig auch in anderen Texten des Diskurses in ähnlicher Form wiederfinden und eine |stabile Abgrenzung des zu definierenden Begriffs/Sachverhalts von diesen Vergleichswerten stimulieren|,

- $\quad$ mit repräsentativer Grundfunktion.

Typ 1a wurde in Kapitel 6.2.2.1 und 6.2.2.2 an verschiedenen Beispielen mit leichten internen Variationen beschrieben (siehe prototypisch die Belege 2 und 4 in Kap. 6.2.2.1). Die deskriptiven Definitionsformen insbesondere nach dem

\footnotetext{
Beispiel der Apotheken Umschau 05/05 B: 40 (= AU 2005b, im QV unter 8.1.7): „Setzen Sie sich überschaubare, realistische Ziele.“

754 Es geht um den Anspruch einer „Realdefinition“, das ,reale Z Zeichenobjekt, das als pragmatische Ursache der Zeichenbildung angesehen werden kann (vgl. das Zeichenmodell von Peirce, das in Kap. 2.2.2 beschrieben wird), adäquat definitorisch zu repräsentieren. Auf diese Funktion wird im Fließtext in diesem Kapitel noch eingegangen.
} 
Muster genus proximum - differentia specifica sind nach Brinker/Cölfen/Pappert (82014: 64) „besonders charakteristisch[e]“ für „Lexikonartikel, wissenschaftliche Abhandlungen“. Deskriptionen dieses Typs gelten fachkulturell als geeignete Vertextungsmuster, um einen Sachverhalt sachlich und neutral von verschiedenen Seiten aus zu beleuchten (ebd.), auch wenn es mittlerweile ebenfalls deutliche Kritik an Ansätzen der klassischen Terminologielehre und systemlinguistisch geprägten Fachlexikografie gibt, die an dieses klassische aristotelische Muster anknüpfen (vgl. Kap. 4.2.4).

In Hand- und Lehrbüchern sowie fachlichen Übersichtsartikeln und einigen Originalarbeiten ${ }^{755}$ findet man meist Typ 1b) mit mehreren präsentierten Definitionen, von denen eine aber in hervorgehobener Weise dargestellt wird. ${ }^{756}$ Die anderen nicht hervorgehoben präsentierten Definitionen in Hand- und Lehrbüchern ergeben streng genommen einen weiteren Untertyp, dem eine geringere transtextuelle Diskurswirkmacht zukommt.

Typ 1c) ähnelt den anderen beiden Typen, was damit zusammenhängt, dass sich die Online-Enzyklopädie Wikipedia und Online-Nachschlageportale wie NetDoktor oder Onmeda am Lexikonstil orientieren. Bei Wikipedia konnte zudem gezeigt werden, dass in den Artikelversionen ab dem Jahr 2009 bis 2016 der erste Definitions-Abschnitt des Wikipedia-Eintrags auf der Definition des Klinischen Wörterbuchs Pschyrembel (261. Auflage, 2007, siehe Beleg 4 in Kap. 6.2.2.1) basierte und dadurch auch die Definitionsentfaltungsform übernommen wurde (vgl. Kap. 6.2.3.2).

Das auf Aristoteles zurückgeführte Muster der Angabe des nächsthöheren Gattungsbegriffs (genus proximum) und der ,spezifizierenden Artkennzeichnung (=differentia specifica)“ (Bußmann ${ }^{3}$ 2002: 148; vgl. auch Roelcke ${ }^{3} 2010$ : 62) ähnelt diesen Mustern des Typs-1 von der Form her sehr, ohne dass damit alle strengen Gütekriterien, die mit dieser Definitionsform terminologisch und philosophiegeschichtlich einhergehen, in den Belegen strikt eingehalten werden (siehe Kap. 4.2.3 und 4.2.4). Die Beschreibung erfolgt meist aus einer synchron-statischen Perspektive auf `Zustände`: Es werden aus der Rückschau die somatischen und psychisch-sozialen Symptome in überwiegendem Maß als Endpunkte von in der Regel pejorativen transformatorischen Vorgängen, Zustandswechseln und teilweise Handlungen, die zu diesem Endpunkt führen, beschrieben (dauernden

755 Bei Originalarbeiten oft in Verbindung mit aufzählenden, operationalen Definitionen, siehe Typ 3.

756 Die Hervorhebung erfolgt typo- und/oder topografisch oder durch spezielle Formulierungen (z. B.: die am häufigsten zitierte Beschreibung von Burnout geht auf [...] zurück: [...]; Im Kontext dieser Übersicht soll die Definition des Burnout-Syndroms herangezogen werden, die sich in der Literatur mit Abstand am häufigsten findet, vgl. dazu Kap. 6.2.2.4 und 6.2.4.2). 
Gefühl der Überforderung, Zustand emotionaler Erschöpfung, reduzierter Leistungsfähigkeit, Dehumanisierung).

In diesen TYP-1-Definitionen wird der Ausdruck/Gegenstand BURNOUT durch Bezug auf ein (terminologisches) Definiens-Nomen höherer Abstraktion (Syndrom, Zustand, Konzept, Krise, (Risiko-)Zustand) einer übergeordneten Kategorie und teilweise auch schon einem Bereich zugeordnet (Syndrom verweist auf den med./klinisch-psychologischen Bereich). Dieses Definiens-Nomen wird im Anschluss meist durch adjektivische oder genitivische Konstruktionen spezifiziert (Syndrom emotionaler Erschöpfung), weitere Kernmerkmale (in diesem Typ-1 häufig insgesamt drei) werden nach ähnlichem Muster auf begrenztem Raum aufgelistet und charakterisiert, und es wird entweder indefinit-generisch (man) formuliert oder die typischen PERSOnEngruppen, die diese Merkmale zeigen, und Zusammenhänge, in denen sich diese PERSONENGRUPPEN befinden, werden benannt (besonders in helfenden Berufen). Teilweise werden zudem am Rande Entstehungsbedingungen und Phasen expliziert (z. B. inf. Diskrepanz zw. Erwartung u. Realität; Endzustand eines Prozesses von idealist. Begeisterung über Desillusionierung, Frustration und Apathie). Diese weisen intertextuelle Bezüge zu Erklärungsmodellen (wie z. B. der Gratifikationskrise) auf, die andernorts im fachlichen Diskursstrang im Kontext von >Burnout erforscht werden. ${ }^{757}$ Sie bestimmen den Gegenstand demnach direkt als intersubjektiv-kollektives Phänomen, was dem Anspruch auf neutrale, objektive Sachverhaltskonstitution entspricht. Beschreibende Definitionen dieses Typs lassen sich auch gut mit dem begrenzten Platz in Fachlexika, Hand- und Lehrbücher vereinen und entsprechen dadurch zusätzlich dem definitorischen Anspruch, dass Definitionen sich auf wesentliche Faktoren beschränken sollten. Die informierende Hauptfunktion von Texten in medizinischen und psychologischen Fachlexika sowie der Einsatz von sprachlichen Mitteln zur Untermauerung der Gültigkeit, Validität und Intersubjektivität der Definitionen und der Adäquatheit zwischen Definiens und Definiendum (vgl. vor allem Kap. 6.2.2.1 und Kap. 6.2.3.1 und 6.2.4.2) legen nahe, dass in diesen Publikationsorganen ein ontologischer Denkhorizont vorherrscht, der von den Definitionen eine Aussage über einen Sachverhalt im „Reich der Wirklichkeit“ erwartet (vgl. Felder 2006a: 167). Dadurch drückt sich in diesen Definitionen eine repräsentative Grundfunktion aus.

757 Zum Beispiel ist der Kern des Konzepts der „Gratifikationskrise“ ebenfalls eine Diskrepanzerfahrung „,zwischen Verausgabung und Belohnung“(Klein/Grosse Frie/Blum et al. (2010: 375), in PPmP, im QV unter 8.1.4), und die Phasen der Begeisterung, Desillusionierung, Frustration und Apathie findet man in ähnlicher Weise auch in anderen häufig zitierten Phasenmodellen (vgl. dazu Kap. 6.2.4.2). 
Teilweise präsentieren die Hand- und Lehrbücher, ähnlich wie in fachlichen Übersichts- und Originalarbeiten, mehrere Definitionen verschiedener Autorinnen und Autoren nebeneinander, die jedoch alle für sich diesen sachgemäßem Realitätsbezug beanspruchen. Wenn in einem etabliert-anerkannten klinischen Fachwörterbuch wie dem Pschyrembel hingegen nur eine Definition ohne Hinweis auf die Urheberschaft erscheint, so könnte diese Definition von einigen Leser/innen als aktueller fachlicher Konsens bzw. deklarierte „endgültige Meinung “ interpretiert werden (vgl. Peirce, CP 8.148), auf deren Basis institutionell Entscheidungen getroffen werden können (vgl. Kap. 4.3.2). Dieser Effekt wird verstärkt, wenn in weiteren Fachlexika, Hand- und Lehrbüchern und Übersichtsarbeiten dieselbe Definitionsvariante erscheint oder wenn diese bei der Präsentation mehrerer Definitionsvarianten typografisch (durch Fettdruck oder Einrahmung) und/oder lexikalisch (die anerkannteste oder verbreitetste Definition ...) hervorgehoben wird (vgl. Kap. 6.2.4.2).

Aus diachroner Perspektive konnte am Beispiel des Burnout-Diskurses gezeigt werden, dass sich die Definition nach Maslach/Jackson (1981/1984), die ursprünglich dem deskriptiven Definierens-Typ $1 b^{758}$ verbunden mit einer operationalen Definition des Typs 3a entspricht, sich gegenüber anderen Definitionsvarianten als dominante Definition bis heute behauptet (vgl. Kap. 6.2.4.2). Dies könnte daran liegen, dass diese Definitionsentfaltung neutral-objektorientiert formuliert ist, die Wahl des Hyperonyms (Syndrom) eine klare Zuordnung zum medizinischtherapeutischen Bereich vornimmt und die deskriptive Entfaltung von drei Kernsymptomen dem Kriterium wesentlicher und nicht ausufernder Spezifizierung entspricht. Die intertextuellen Wiederholungen derselben Definitionsvariante insbesondere in Fachlexika, Hand- und Lehrbüchern verknappen den Diskurs, wie am Burnoutdiskurs gezeigt wurde, maßgeblich (siehe Kap. 6.2.4.2). Sie ebnen diskursiv den Weg hin zu einer allgemein akzeptierten Definition, dadurch dass divergierende Konzepte im Diskurs weniger sichtbar werden und dadurch der Eindruck von Übereinstimmung entsteht. Eine solche Diskursposition erhöht die Chance, dass diese Definitionsvariante gesellschaftlich-institutionell eingebunden wird (wie z. B. im Mai 2019 in der 11. Fassung der ICD der WHO) ${ }^{759}$, wodurch sie schließlich deklarative Kraft erhalten könnte (vgl. Beleg 12 in Kap. 6.2.2.2).

758 Nur mit dem Unterschied, dass Maslach/Jackson keine anderen Burnout-Definitionen diskutieren, sondern direkt ihre Definition vorstellen und die dahinter stehende Operationalisierung beschreiben (Maslach/Jackson 1981/1984).

759 „Burn-out“ ist dort unter dem Schlüssel „QD85“ mit drei Symptomen, die zur dreifaktoriellen Definition von Maslach/Jackson (1984) und späteren Lexikondefinition im Burnout-Diskurs große Ähnlichkeit aufweisen, im Kapitel „Problems associated with employment or unemployment“ als Zusatzschlüssel eingebunden. Siehe dazu den Eintrag in der ICD-11 der WHO (= WHO 2019a/b) 
Die Typen 1a)-1c) weisen des Weiteren stabil sprachliche Marker bzw. Stimuli an der Diskursoberfläche auf (sprachliche Mittel der Quantifizierung, Gradierung, Negation), durch die der beschriebene Zustand im Vergleich zu ähnlichen inferierten 'gesunden', 'normalen' oder auch 'erwünschten' physischen und psychischen Zuständen ein reduziertes bzw. negiertes oder quantifiziertes bzw. gesteigertes Maß präsentiert (z. B. chronische Erschöpfung, emotional überanstrengt, reduzierte Leistungsfähigkeit, negative, gefühlslose und zynische Einstellungen). Zeichenkombinationen, die diese Inferenzen stimulieren, verweisen auf ein gemeinsames generisches Wissen, was - bezogen auf einen >durchschnittlich gesunden Menschen eines bestimmten Alters ‘ (vgl. die `Speziesnorm ‘ bei Rachidi 1989: 99 und Kap. 6.2.4.1) - als 'normale' >Erschöpfung` oder als 'normale' Häufigkeit für Infektionen der Atemwege zu gelten hat. Auch bei der Beschreibung PSYCHISCHER ZUSTÄNDE, PERSONELLER EIGENSCHAFTEN und VERHALTENSAUFFÄLLIGKEITEN kommen durchschnittliche menschliche und gruppenspezifische Vergleichswerte und gesellschaftliche und kulturelle Erwartungsnormen mit ins Spiel (vgl. Kap. 6.2.4.1 zu den dort beschriebenen Ideal- und Tauglichkeitsnormen, die in Konflikt zueinander geraten und dadurch zum Sумртом werden können). Diese Inferenzen findet man zum einen punktuell und transtextuell in den Definitionen dieser Typen 1a bis c, aber ebenso in anderen Definitionstypen des Burnout-Diskurses, die im Folgenden noch beschrieben werden. Diese konstante transtextuelle Stimulierung ähnlicher Inferenzen birgt ein hohes diskursives Unifizierungspotenzial in dem Sinne, dass bestimmte Merkmale des Konzepts `Burnout` in Abgrenzung zu diesen Inferenzwerten über verschiedene Definitionen hinweg erscheinen und dadurch gesamtdiskursiv definitorische Kraft erhalten (vgl. Kap. 6.2.4.1).

Deskriptive Definitionsentfaltungstypen zeigen sich fachextern nicht nur in den Online-Nachschlagewerken, sondern auch in medialen Textsorten, dann gerne als Definitionen, die Fachleuten zugeschrieben werden oder aus Fachwörterbüchern zitiert werden (siehe z. B. SPIEGEL ONLINE, 12.02.2013 (= Lobo 2013); Süddeutsche Zeitung, 29.11.2008: V2/9 (= SZ 2008a)). Sie folgen des Weiteren als Antwortsequenzen in dialogisch aufgebauten Texten (siehe die Belege D, E und F in Kap. 6.2.3.1). In den Medientexten stehen sie aber meist nicht in solch hervorgehobener Anfangsposition wie in den Fachlexika, Hand- und Lehrbüchern, sondern in verkürzter Form kombiniert mit anderen (z. B. narrativen) Definitionsentfaltungsformen (siehe z. B. Beleg 4 in Kap. 6.2.3.1), die im folgenden Typ diskursiver Praxis des Definierens beschrieben werden:

unter: https://icd.who.int/browse11/l-m/en\#/http://id.who.int/icd/entity/129180281, zuletzt abgerufen am 30.12.2019; im QV unter 8.1.3). 


\section{Typ 2a: Eine sich narrativ entfaltende $>$ EREIGNIS $\iota^{760}$-Definition,}

- $\quad$ die /den zu definierenden Ausdruck/Begriff/Sachverhalt in Form eines (professionellen) (Diagnose-)Kommentars ${ }^{761}$ verallgemeinernd einer singulären Beispiel-Erzählung zuweist|,

- $\quad$ die in der Beispielerzählung |das Individuum durch verschiedene Merkmale (Beruf, Alter, persönliche Eigenschaften) charakterisiert| und |durch die Schilderung von Begleitumständen situiert| und |Handlungen sowie plötzlich oder allmählich auftretende Merkmale/Verhaltensweisen des Individuums in zeitlich geordneter Abfolge erzählt|,

- die |sich von durchschnittlichen menschlichen und gruppenspezifischen Vergleichswerten/Handlungen sowie gesellschaftlichen und kulturellen Erwartungsnormen unterscheiden| und

- die |sich auch in anderen Texten des Diskurses in ähnlicher Form wiederfinden und dadurch eine stabile Abgrenzung des zu definierenden Begriffs/Sachverhalts von diesen Vergleichswerten stimulieren|,

- $\quad$ die |zu Beginn eines fachexternen Medientextes präsentiert wird|

- $\quad$ mit repräsentativ-deklarativer Grundfunktion.

\section{Typ 2b: Eine sich narrativ-kontrastiv entfaltende $>$ Ereignis $\triangleleft / /$ Vorgangs $\iota^{762}$ - Definition,}

- $\quad$ die /den zu definierenden Ausdruck/Begriff/Sachverhalt in Form eines (professionellen) (Diagnose-)Kommentars verallgemeinernd einer singulären oder mehreren generischen Beispiel-Erzählung/en zuweist|,

- die in den Beispielerzählungen |die typischen Personengruppen durch verschiedene Merkmale (Beruf, Alter, persönliche Eigenschaften) charakterisiert| und |durch die Schilderung von Begleitumständen situiert| und |den typischen Personengruppen Handlungen sowie Merkmale/Verhaltensweisen eines Ausgangszustands-1 und eines dazu im Kontrast stehenden Endzustands-2 zuschreibt|,

- wobei die Merkmale/Verhaltensweisen des Ausgangszustands-1 meist über den inferierten durchschnittlichen menschlichen und gruppenspezifischen Vergleichs-

760 `Ereignis` wurde in Kapitel 6.2.2.3 als eine Entwicklung, die sich „auf der Zeitachse“ vollzieht, bestimmt (Müller 2007: 82). `Ereignissen` kommt im Vergleich zu ıVorgängen das semantische Merkmal des 'Einmaligen' zu (vgl. Brinker/Cölfen/Pappert ${ }^{8} 2014:$ 60). Die narrativen Definitionen in medialen Magazinberichten erzählen meistens zunächst Individualbeispiele, die erst in einem zweiten Schritt verallgemeinert werden. Daher wurden diese Definitionen mit dem Metakonzept `Ereignis benannt.

761 Meist erscheint der (Diagnose-)Kommentar anaphorisch nach der Beispielerzählung, teilweise aber auch kataphorisch durch einen einleitenden Absatz vor der Beispielerzählung.

762 `Vorgängeく beschreiben im Vergleich zu `Ereignissen` 'regelmäßige' Abläufe (vgl. Brinker/ Cölfen/Pappert ${ }^{8}$ 2014: 62). Wenn sich im Burnout-Diskurs eine Definition anhand generischer Beispiele narrativ entfaltet, so wird sie daher als Vorgangs-Definition typisiert. Nach von Polenz sind `Vorgänge` des Weiteren mit den Merkmalen 'unwillkürlich', 'ohne Absicht' verbunden (von Polenz ${ }^{3}$ 2008: 161f., siehe auch Kap. 6.2.2.3). 
werten/Handlungen und die Merkmale/Verhaltensweisen des Endzustands-2 unter den durchschnittlichen Vergleichswerten liegen

- und diese Vergleichswerte |sich auch in anderen Texten des Diskurses in ähnlicher Form wiederfinden und dadurch eine stabile Abgrenzung des zu definierenden Begriffs/Sachverhalts von diesen Vergleichswerten stimulieren|,

- $\quad$ die |zu Beginn eines fachexternen Medientextes präsentiert werden|,

- mit repräsentativ-deklarativer Grundfunktion.

\section{Typ 2c: Eine sich referierend entfaltende >EREIGNIS <-Definition,}

- $\quad$ die /den zu definierenden Ausdruck/Begriff/Sachverhalt in Form eines professionellärztlichen Diagnose-Kommentars als Akt einer verallgemeinernd-repräsentativen/ deklarativen Erklärung einem singulärem Beispiel-Bericht zuweist|,

- $\quad$ die in dem Beispielbericht |den Zustand des Individuums durch Merkmale und Anzeichen, die durch Untersuchung festgestellt und vom Individuum geschildert wurden, charakterisiert| und |durch die Wiedergabe der vom Individuum geschilderten Begleitumstände situiert| und |Handlungen sowie auftretende Merkmale/Verhaltensweisen aus der Schilderung des Individuums wiedergibt|,

- die |sich von durchschnittlichen menschlichen (biologischen und psychischen) und gruppenspezifischen Vergleichswerten/Handlungen sowie gesellschaftlichen und kulturellen Erwartungsnormen unterscheiden/ und

- die |sich auch in anderen Texten des Diskurses in ähnlicher Form wiederfinden und dadurch eine stabile Abgrenzung des zu definierenden Begriffs/Sachverhalts von diesen Vergleichswerten stimulieren|,

- $\quad$ die |den Haupttext der Textsorte Kasuistik nach dem Abstract eröffnet| // die lin einen fachlichen Übersichtsbeitrag eingebettet ist|,

- mit repräsentativ-deklarativer Grundfunktion.

\section{Typ 2d: Eine sich referierend entfaltende >Vorgangs $`$-Definition}

- $\quad$ die |den zu definierenden Ausdruck/Begriff/Sachverhalt einem Phasen-Modell repräsentativ zuweist| und |auf typische Personengruppen generisch referiert|,

- die in den Phasenbeschreibungen /die typischen Personengruppen durch verschiedene Merkmale (Beruf, Alter, persönliche Eigenschaften) charakterisiert| und |durch die Schilderung von Begleitumständen situiert| und |den Personengruppen typische Handlungen sowie Merkmale/Verhaltensweisen für die verschiedenen Phasen zuschreibt|, - die |sich von durchschnittlichen menschlichen (biologischen und psychischen) und gruppenspezifischen Vergleichswerten/Handlungen sowie gesellschaftlichen und kulturellen Erwartungsnormen graduell je nach Phase unterscheiden/ und

- $\quad$ die sich auch in anderen Texten des Diskurses in ähnlicher Form wiederfinden und dadurch eine stabile Abgrenzung des zu definierenden Begriffs/Sachverhalts von diesen Vergleichswerten stimulieren|,

- die in einen fachlichen/fachexternen Übersichtsbeitrag eingebettet ist,

- mit repräsentativer Grundfunktion.

Die narrativen Definitionsentfaltungsmuster 2a und 2b erscheinen nur im fachextern medialen Diskursstrang. Typ 2 a wurde in Kapitel 6.2.3.1 an verschiedenen 
Belegen beschrieben (siehe die Belege 2, 3, 5 und 6 und $7^{763}$ ). Eine narrative Definitionsentfaltung, die man insbesondere in medialen Magazinberichten findet: Bei dieser Entfaltungsart werden die erzählten Merkmale des zu definierenden Phänomens und dessen Entwicklungsbedingungen als Ereigniskette über die Zeit anhand eines Fallbeispiels (entweder eines Individuums ${ }^{764}$ oder Vertreters einer Personengruppe, z. B. Lehrer/in) entwickelt. Durch einen der Erzählung vorausgehenden kurzen Einleitungsabschnitt oder nachfolgenden Kommentar wird der Erzählung der Ausdruck/Gegenstand `BURNOUT« zugeordnet. Die Autorinnen und Autoren von Texten in Zeitungen und populärwissenschaftlichen Zeitschriften sind an den Grundsatz der ,wahrheitsgemäße[n] Berichterstattung“ gebunden. ${ }^{765}$ Ihre Erzählungen sind faktual und die Leser/innen gehen davon aus, dass die präsentierten Erzählinhalte „der Welt entsprechen“ (Searle 1980: 84, vgl. Kap. 4.3.2). Dadurch erscheint die Definitionserzählung einerseits als repräsentativ-emotive Sprachhandlung. Durch den der Narration folgenden Deutungskommentar, der meist von einer Person aus dem medizinischen oder therapeutischen Bereich stammt und als „Diagnose“ eine Krankschreibung bewirken kann, erscheinen die geschilderten Symptome andererseits in einem deklarativen Licht. Denn sie werden dadurch zumindest innerhalb der Erzählung mit einem Diagnoseausdruck verknüpft, der die Kraft besitzt, gesellschaftliche Tatsachen zu schaffen. Selbst wenn in Medientexten nach der Präsentation des narrativen Fallbeispiels und Diagnosekommentars die Kritik formuliert wird, dass >Burnout strenggenommen keine „Diagnose“, sondern ein „Risikozustand“ sei (siehe dazu auch den Definitionsentfaltungs-Typ 5), so besitzt diese narrative Definitionsform durch ihre Praxisnähe und häufige Texteröffnungsposition diskursive Wirkmacht. Dies hängt sicher auch damit zusammen, dass in diesen Definitionen, anders als in den Definitionen des Typs 1, das Definiendum >BURNOUT< zunächst anhand eines Beispiels konkretisiert und danach erst durch die Diagnose verallgemeinert wird. Dieser Wechsel vom individuellen Erleben und Auftreten der körperlichen und psychischen Merkmale (als Sinzeichen) zur verallgemeinernden Interpretation vor dem fachlichen Hintergrund ähnlicher Fälle (als medizinische

763 In Beleg 7 in Kap. 6.2.3.1 ist Typ 2 nochmals dadurch variiert, dass hier aus der IchPerspektive erzählt wird. In den meisten Beispielen dieses Typs, die in der vorliegenden Arbeit analysiert wurden, erfolgt die Erzählung jedoch aus der Er-Perspektive. Weitere Beispiele aus dem Korpus, die >Burnout nach Typ 2-a definieren sind z. B.: PH 1/2016: 18-21 (= Schönberger 2016a, im QV unter 8.1.7); G\&G 11/2005: 12-19 (= Kraft 2005, im QV unter 8.1.7).

764 Die individuellen Beispiele werden aber meistens auch als Vertreter bestimmter Gattungen präsentiert, so zum Beispiel in Beleg 5 in Kap. 6.2.3.1 „Bernd Hinze“ (ein Pseudonym), der als „Werbetexter“ arbeitet, oder in Beleg 6 „Frauke B.“, die als Malerin klassifiziert wird.

765 Vgl. zum Beispiel den Ethik-Kodex des deutschen Fachjournalistenverbands: https://www. dfjv.de/beruf/journalismus-als-beruf/berufsethik, zuletzt eingesehen am 30.12.2019. 
Legizeichen) ist typisch für den medizinischen und klinisch-psychologischen Fachbereich und untermauert dadurch die Geltung dieser Definition als typische Praktik dieser Fachbereiche (vgl. Kap. 3.1).

Typ 2b erscheint ebenfalls vor allem im fachexternen Mediendiskurs. Statt der Präsentation einer Ereigniskette werden der Anfangs- und Endzustand meist anhand von Beispielvertretern typischer Berufsgruppen (die junge Lehrerin, Psychologin, Arbeitnehmer) kontrastierend geschildert (siehe z. B. Beleg 1 in Kap. 6.2.3.1). ${ }^{766}$

In Typ 2c wird auch eine individuelle Geschichte im Rahmen der Fachtextsorte Kasuistik wiedergegeben (siehe z. B. Beleg 8 in Kap. 6.2.2.2). Dass hier von einer referierenden statt narrativen Themenentfaltung gesprochen wird, geht auf die stilistische Unterscheidung zurück, dass die fachliche Fallschilderung ergebnisorientiert Symptome und Lebensumstände berichtet, die im Rahmen einer Anamnese erfragt bzw. von der Patientin/dem Patienten berichtet wurden. Das Referierte wird im Vergleich zu den medialen Erzählungen nicht mit Spannungsbögen und Kontrastlinien narrativ ausgestaltet (vgl. zur Unterscheidung dieser Themenentfaltungstypen Kap. 6.2.2.3, 6.2.2.4 und 6.2.3.3).

Typ 2d schließlich ist ebenfalls stärker referierend als erzählend. Es werden typische Phasen der Burnout-Entwicklung mit allgemein-generischer Referenz (z. B. über den Gebrauch des Indefinitpronomens man) oder durch den Bezug auf besonders ,betroffene' Personengruppen, wie z. B. Ärzte und Therapeuten (vgl. im Dt. Ärzteblatt, Will 2014: 2, im QV unter 8.1.4) mit den für diese Phasen charakteristischen Merkmalen im komprimierten Nominalstil beschrieben. Diese Definitionsentfaltung ähnelt der Beschreibung eines sich in dieser Abstufung typischerweise wiederholenden Vorgangs, der mit einem repräsentativen Anspruch geäußert wird, dass diese Phasen sich in der ,Wirklichkeit‘ in dieser Weise vollziehen.

Die spezifizierende Abgrenzung vom ,normalen` bzw. ,gesunden` Verhalten und Erleben oder anderen sachverwandten Konzepten erfolgt in narrativen Definitionsformen des fachexternen Diskursstrangs auf der konkreten Handlungsebene am Einzelbeispiel, expliziert durch eine große Bandbreite sprachlicher Mittel: sowohl über Fokuspartikeln, Intensitätspartikeln und Abtönungspartikeln als auch über Adjektive und Adverbien, die die beschriebenen Sachverhalte quantifizieren oder steigern sowie über substitutive und konzessive Konnektoren, durch die Komponenten von >Burnout` von anderen `Zuständen`, `Merkmalen`, `Bedingungen` unterschieden werden (vgl. Kap. 6.2.3.1). Durch diese Bandbreite

766 Weitere Beispiele aus dem Korpus: faz.net 08.03.2010 (= Meck 2010, im QV unter 8.1.6); AU 11-2012-A: 22-26 (= Schweiger 2012, im QV unter 8.1.7). 
erfüllen narrative Definitionen weniger als Definitionen in Fachlexika und Handbüchern des Typs 1 den Anspruch auf angemessene Umgrenzung und Konzentration auf wesentliche Faktoren. Diese Abgrenzungs- und Bestimmungsmerkmale werden in deskriptiven Definitionen im fachinternen Diskursstrang zudem in sprachökonomisch-komprimierterer und weniger konkreter Form innerhalb unpersönlicher hypotaktischer Attribut- und Nominalkomplexe ausgedrückt (vgl. Kap. 6.2.2.1). In beiden Definitionstypen verweisen diese sprachlichen Mittel der Quantifizierung, Gradierung und Negation jedoch dennoch auf ähnliche sozial-individuelle Erwartungen (z. B. in Bezug auf die Arbeitsleistung und Einstellungen zur Arbeit) und durchschnittlich-biologische Normwerte (z. B. in Bezug auf Erschöpfungsmaße). Das zeigt sich, wenn man diese sprachlichen Marker, die als Symptome fungieren, intertextuell miteinander vergleicht: Z. B. wird im Dt. Ärzteblatt von „Leistungsminderung“ gesprochen; ${ }^{767} \mathrm{im}$ medialen Diskurs findet man ein ähnliches Symptom, allerdings in gesteigerter Form: „Die eigene Arbeit wird als wirkungslos erlebt [...]“768; In der Zeitschrift „Der Psychotherapeut“ wird die Beschreibung „ausgeprägte Erschöpfungssymptomatik“ verwendet; ${ }^{769}$ im medialen Diskurs wird ein ähnliches Symptom durch die folgende Aussage realisiert: [...] „Ich hatte keinen Antrieb zu gar nichts, alles war mir zu viel“;770 eine direkte ,Übersetzung‘ von Fach- in Alltagssprache findet man schließlich in folgendem Zitat, in dem der Psychotherapeut A. Bergner auf die Frage der ZEITReporter/innen „Wie kann ich feststellen, ob ich unter Burn-out leide?“ antwortet:

Bergner: Erstes Symptom ist die emotionale Erschöpfung. Man kann sie selbst diagnostizieren. Sätze wie „Ich kann nicht mehr“ oder „Es macht alles keinen Sinn“ sind klassisch für emotional erschöpfte Menschen. Zweites Symptom ist die sogenannte Depersonalisation. Die Betroffenen ziehen sich zurück. Das dritte Symptom ist die Leistungsabnahme. Erst wenn alle drei Symptome erfüllt sind, lässt sich die Diagnose Burn-out stellen. [.... $]^{771}$

Des Weiteren werden von Fachleuten entwickelte Phasen-Modelle medial aufgegriffen und narrativ verarbeitet: Zum Beispiel werden die Symptome ,vermehrtes Engagement für Ziele“ in „der Anfangsphase“ und der „Abbau“ der „kognitiven Fähigkeit“ in einer späteren Phase (vgl. Burisch ${ }^{5} 2014:$ 25-29) in medialen Maga-

767 Siehe z. B. bei Berger/Falkai/Maier 2012: 213, Dt. Ärzteblatt, im QV unter 8.1.4.

768 Siehe, FAZ, 19.4.2014, (= Weiguny/Nienhaus 2014a, im QV unter 8.1.6).

769 Siehe in der Fachzeitschrift „Der Psychotherapeut“ (= Weimer/Kraus 2011: 242, im QV unter 8.1.4).

770 Auf faz.net, 8.3.2010, (= Meck 2010, im QV unter 8.1.6).

771 Für weitere Beispiele intertextueller Bezüge zwischen fachlichen und fachexternen Texten siehe Kap. 6.2.4.2. 
zinberichten und ratgebenden Aufklärungstexten entweder chronologisch oder durch kontrastive Zustände geschildert (siehe die Definierens-Typen 2a und 2b).

Dennoch ist $\mathrm{zu}$ bedenken, dass in fachlichen Kontexten beim Gebrauch von Ausdrücken wie Belastung, Beanspruchung, Stress und Ermüdung oder Erschöpfung andere Referenzzustände und Gebrauchszusammenhänge als in fachexternen Kontexten sowie terminologische Abgrenzungen eine Rolle spielen. Denn Mediziner/innen und Psychologinnen/Psychologen besitzen andere Wissensgrundlagen als Laien (vgl. Busch 1999: 104ff.), manche Termini, wie z. B. Belastung und Beanspruchung sind genormt ${ }^{772}$ oder beruhen auf Operationalisierungen $^{773}$, und „probabilistisch formulierte Wirkhypothesen“ von fachlicher Seite (Rau/Henkel 2013: 792) ${ }^{774}$ werden fachextern häufig zu Wirkzusammenhängen.

Da im Bereich Medizin/Psychologie Symptome wie `Erschöpfung` oder \Leistungsminderung، neben physiologisch-messbaren Faktoren und Beobachtungsmerkmalen jedoch immer auch auf den Auskünften der ,betroffenen“ Personen zu ihrem Erleben oder auf Aussagen von dritten Personen zum deren Verhalten beruhen, sind Überschneidungen der Fach- und Alltagssemantik in diesem Fachund Praxisbereich von Grund auf angelegt (vgl. dazu auch Kap. 3.2). ${ }^{775}$ Diese Überschneidung findet man in besonderer Weise auch im folgenden Typ einer diskursiven Praxis de Definierens:

772 Vgl. DIN EN ISO 10075-1:2000:11 und Nachreiner (2012: 9) sowie im Überblick Kap. 5.2. 773 So schreibt Rigotti im „Lexikon der Psychologie - Dorsch“, dass ıpsychische Belastungen meist über „Zeitdruck“ und „Entscheidungsspielraum“ operationalisiert werden (Rigotti 2019). 774 In der Fachzeitschrift „Der Nervenarzt“, im QV unter 8.1.4.

775 Auch Schuster betont, dass die ,alltägliche Sicht auf den Menschen und psychiatrische Fachsprache“ heute in einem „produktiven, wechselseitigen Abhängigkeitsverhältnis“ stehen (Schuster 2010: 13, vgl. auch Kap. 5.3.1). In fachlichen Definitionen zum Burnout-Phänomen scheinen die in den definierenden Propositionen verwendeten Ausdrücke jedoch nicht durchgängig an die deutschsprachige medizinisch/psychologische Fachsprache terminologisch rückgebunden zu sein. So bedeutet „Depersonalisation“, im Burnoutdiskurs für eine zynisch-abwertende Haltung gegenüber anderen Personen (bei der Arbeit) verwendet, in der deutschen medizinischen Fachsprache eigentlich, dass „das Erleben der persönlichen Einheit im Augenblick oder der Identität über den Lebenszeitlauf gestört ist. “Siehe dazu den Eintrag im klinischen Wörterbuch Pschyrembel zu Depersonalisation (= Margraf 2016, im QV unter 8.1.2). Diese terminologische Ungenauigkeit kann man auch bei der Bezeichnung Überlastung vermuten, da die in der Arbeitspsychologie üblichen, sogar in DIN-Normen (z. B. DIN EN ISO 10075-1:2000:11) verwendeten Termini Belastung und Beanspruchung in vielen Burnout-Definitionen nicht verwendet werden (vgl. zu den fachkulturellen, fachpolitischen, sozialpolitischen und gesellschaftlichen Rahmenbedingungen des Burnout-Diskurses auch Kap. 5.2. dieser Arbeit). Diese terminologische Ungenauigkeit wird im Burnout-Diskurs von fachlicher Seite aus von verschiedener Seite aus kritisiert (z. B. bei Rook (1998: 99), die „eine fehlende Genauigkeit (Vagheit) hinsichtlich der Bedeutungszuschreibungen innerhalb verschiedener Theorienzusammenhänge“ beklagt (vgl. zu dieser Kritik auch Kap. 4.2.2). 


\section{Typ 3a: Eine sich aufzählend-deskriptiv entfaltende, nach wissenschaftlichen Metho- den operationalisierte Zustandsdefinition,}

- die /den zu definierenden Sachverhalt/Begriff/Ausdruck einer Liste von Einzelfragen/ Einzelaussagen (Fragebogenitems) zuweist|,

- die |charakteristische Attribute/Merkmalsausprägungen des zu definierenden Zustands operationalisieren|, dadurch dass sie |Items entwerfen, die für das Verhalten und Erleben von Personen, die sich in diesem Zustand befinden, typisch oder untypisch sind|,

- die |sich (implizit) auf durchschnittliche menschliche und gruppenspezifische Vergleichswerte und gesellschaftliche und kulturelle Erwartungsnormen beziehen|, die |sich auch in anderen Texten des Diskurses in ähnlicher Form wiederfinden|,

- die laufgrund empirisch-statistischer Reliabilitäts- und Validitätstests und einer methodisch kontrollierten Auswertung durch Fachleute postuliert, dass die operationalisierten Items den empirisch beobachteten Merkmalsausprägungen entsprechen| und |direktiv die einzelnen Versuchspersonen dazu auffordert, die Iteminhalte mit dem eigenen Erleben und Verhalten zu vergleichen und ihnen (graduell) zuzustimmen oder diese abzulehnen|,

- $\quad$ in einer fachlichen Originalarbeit als einziges Messinstrument oder neben anderen Messinstrumenten sachverwandter Begriffe/Sachverhalte verwendet,

- mit repräsentativer Grundfunktion.

Typ 3b: Eine sich aufzählend-deskriptiv entfaltende alltagsweltlich operationalisierte Zustandsdefinition,

- $\quad$ die |den zu definierenden Sachverhalt/Begriff/Ausdruck einer Liste von Einzelfragen/ Einzelaussagen (Fragebogenitems/Punkten einer Symptomliste) zuweist|,

- die |charakteristische Attribute/Merkmalsausprägungen des zu definierenden Zustands operationalisieren|, dadurch dass sie |Items entwerfen, die für das Verhalten und Erleben von Personen, die sich in diesem Zustand befinden, typisch oder untypisch sind|,

- die |sich (implizit) auf durchschnittliche menschliche und gruppenspezifische Vergleichswerte und gesellschaftliche und kulturelle Erwartungsnormen beziehen|, die |sich auch in anderen Texten des Diskurses in ähnlicher Form wiederfinden|,

- $\quad$ die |mit einer an den Laien adressierten Auswertungsanleitung postuliert, dass die operationalisierten Items den empirisch beobachteten Merkmalsausprägungen entsprechen| und/oder |direktiv die einzelnen Leser/innen dazu auffordert, die Iteminhalte mit dem eigenen Erleben und Verhalten zu vergleichen, ihnen (graduell) zuzustimmen oder diese abzulehnen und eine auswertende Einordnung aufgrund der Testergebnisse vorzunehmen/Handlungsfolgen daraus abzuleiten|,

- $\quad$ in einem fachexternen ratgebenden Aufklärungstext als Symptomliste oder als einziges Instrument zum Selbsttest für die Leser/innen,

- $\quad$ mit repräsentativ-direktiver Grundfunktion.

Die Typen 3a und b stehen für eine aufzählend-beschreibende Definitionsentfaltung, die eine Reihe oder Liste von Merkmalen bzw. Fragebogenitems präsentiert: Dieser 
Liste wird der Begriff/Gegenstand \BURNOUT` zum Beispiel durch eine eröffnende Frage, Überschrift oder durch eine der Aufzählung vorausgehende Formulierung wie Burnout-Dimensionen sind: [...]/Burnout wurde mit Hilfe [...] erhoben. Hierzu gehören die Skalen [...]“ in einem Entsprechungsverhältnis zugewiesen. ${ }^{776}$ Die Auswertung der Antworten auf die Fragebogenitems ${ }^{777}$ ergibt die Intensität des zu messenden Begriffs/Gegenstands. Diese Definitionsentfaltung findet man zum einen in Originalarbeiten zur Operationalisierung der einzelnen Variablen des zu messenden Phänomens und zum anderen in Zeitungen und populärwissenschaftlichen Magazinen, die Fragebogen zum Selbsttest (in populärwissenschaftlich angepasster methodisch deutlich weniger kontrollierter Form als in den fachlichen Fragebogen) anbieten. Wissenschaftsgeschichtlich spielen hier operationale und ostentative Definitionsformen (,durch Aufzählung geeigneter Beispiele“, vgl. Prechtl 52016: 131 und Kap. 4.2.3) mit hinein. Diese aufzählende Definitionsentfaltung blickt auf den zu definierenden Gegenstand/Begriff ähnlich wie die deskriptive Definitionsentfaltung aus einer synchron-statischen Zustandsperspektive.

Die kommunikative Funktion des Fragebogens ist eine interrogativ-prüfende, direktive (in Bezug auf die Personen, die diese Fragen beantworten sollen) und geht mit einem repräsentativ-ontologischen Geltungsanspruch einher. Es gibt ein beobachtetes Phänomen in der Welt der Wirklichkeit, das zunächst in eine möglichst adäquate fachsprachlich gefasste konzeptionelle Definition und daraufhin in möglichst adäquate sprachliche gefasste messbare Merkmale ,übersetzt' werden soll (vgl. Maslach/Jackson 1984: 134; Kristensen/Borritz/Villadsen et al. 2005: 193 und Kap. 6.2.2.2). ${ }^{778}$ Definitionen des Typs 1a und b können mit diesen operationalen Definitionsformen korrespondieren, wenn Gruppen von Fragebogenitems den Faktoren bzw. Kernmerkmale, die in den Definitionen des Typs 1 charakterisiert sind, zugewiesen werden. Im Burnoutdiskurs haben die deskriptive Definition des Typs 1b nach Maslach/Jackson (1984, siehe Beleg 2 in Kap. 6.2.2.2) mit den drei Kernmerkmalen emotionale Erschöpfung, Depersonalisation und reduzierte Leistungsfähigkeit und das Fragebogeninstrument Maslach

776 In fachexternen Texten erfolgt diese Zuweisung offen für alle Leser/innen. In fachlichen Originalarbeiten wird diese Zuweisung für Fachleute ebenfalls präsentiert und begründet. In der Testsituation schließlich kennen nur die Versuchsleiter/innen diese Zuweisungsrelation zwischen den Fragebogenitems und dem zu messenden `Konzept` Burnout, den Versuchspersonen ist sie verborgen.

777 In fachexternen Texten wird dafür eine Anleitung gegeben. In Testsituationen kennen nur die Versuchsleiter/innen die Methoden der Auswertung.

778 Vgl. dazu den Anspruch auf „Exaktheit“, den Roelcke in Bezug auf Fachwörter beschreibt. Unter „Exaktheit“ sei in diesem Zusammenhang „ein möglichst adäquater Bezug fachsprachlicher Ausdrücke zu den Gegenständen, Sachverhalten und Vorgängen des betreffenden menschlichen Tätigkeitsbereichs zu verstehen“ (Roelcke 32010: 69), siehe auch Kap. 4.2.2 und Fußnote 158. 
Burnout Inventory, dessen Fragebogenitems diese drei Kernfaktoren operationalisieren, weite Verbreitung erfahren (siehe Kap. 6.2.4.2).

In fachexternen Texten findet man in den Überschriften zu derartigen Symptomlisten häufig den Hinweis auf Warnsymptome, und Auswertungsanleitungen enthalten z. B. die folgenden Sätze:

Wenn Sie mehr als zehn Punkte erreichen, sind Sie burnoutgefährdet. Der Test liefert jedoch nur eine grobe Orientierung und kann keine ärztliche Diagnose ersetzen. Im Zweifelsfall sollten Sie sich professionelle Hilfe suchen.

(G\&G, Dossier 2016: 23, im QV unter 8.1.7, Unterstreichung T.S.)

Das Prädikat warnen, das „zwischen den Repräsentativen und Direktiven steht“ (Harras/Winkler/Erb 2004) ${ }^{779}$, kann im Kontext definitorischer Praxis bewirken, dass der gesellschaftliche Druck, eine deklarative Definition für ein Problem wie >BuRnout< zu finden, steigt. Denn durch diese Aufforderung an die Leser/ innen, die Symptome mit dem eigenen Erleben/Empfinden abzugleichen und im Fall einer Übereinstimmung professionelle Hilfe suchen, entsteht eine Erwartungshaltung an das Gesundheitssystem, das auf der Grundlage von deklarierten Diagnosen abrechnet. Diese ‘warnenden' repräsentativ-direktiven Praktiken des Definierens stehen damit in der Mitte zwischen Definierenspraktiken mit rein repräsentativen und Definierenspraktiken mit deklarativen Geltungsansprüchen.

Eine deklarative Funktion und weite Verbreitung, insbesondere über Wikipedia, kommt auch dem folgenden Typ diskursiver Praxis des Definierens zu:

\section{Typ 4a: Eine sich deskriptiv oder über typografische Mittel entfaltende `Zustands ‘-/ >Gattungs<-Definition,}

- die /den zu definierenden Sachverhalt/Begriff/Ausdruck taxonomisch einer (typografisch hervorgehobenen) Begriffs-/Gegenstandsklasse höherer Abstraktion zuweist| und leinem Klassifikationskürzel zuordnet|,

- die im Anschluss |den Ausdruck durch eine paradigmatische Bedeutungsangabe erläutert| und/oder

- die |Hauptattribute, -merkmale charakterisiert|, (die auf eine diskursmächtige deskriptive Definition des Typs 1a zurückgehen)

- die als Teil eines international anerkannten Klassifikationssystem, das gesellschaftlichinstitutionell eingebunden ist, als einzige Definition erscheint // Typ 4b: die in einem Onlineportal mit intertextuellem Bezug auf das international anerkannte Klassifikationssystem neben anderen Definitionen, aber hervorgehoben zu Beginn des Beitrags erscheint,

- mit deklarativer Grundfunktion.

779 Vgl. den Eintrag zu warnen in der Onlineversion des „Handbuchs für deutsche Kommunikationsverben“ unter: https://www.owid.de/artikel/298934 (zuletzt abgerufen am 18.7.2019). 
Diese Definitionsform funktioniert wie eine Definition, die verbindlich Fachausdrücke in einem taxonomischen System normiert (Galinski/Budin 1999: 2185ff., vgl. Kap. 4.2.3 und 4.2.4). Sie kommt dem Ideal am nächsten, dass das Definiendum als Terminus exakt, eindeutig, einheitlich und verbindlich innerhalb eines Begriffssystems bestimmbar sein soll (vgl. Felder 2009a: 42/43; Roelcke 32010: 69ff.). Da >Burnout in der ICD-10 nicht als Haupt-, sondern nur als Zusatzdiagnose verschlüsselt ist, gibt es für >Burnout` in dieser Fassung der ICD keine ausführliche deskriptiv-taxonomische Definition wie für das Diagnosekonzept $>$ Depression ${ }^{780}$ oder $>$ Neurasthenie ${ }^{781}$. In der ICD-11, die ab Januar 2022 gelten soll, ${ }^{782}$ ist >Burnout « nach wie vor nur als Zusatzdiagnose unter den „Factors influencing health status or contact with health services“ als „occupational phenomenon“ gelistet, aber die Symptome sind ausführlicher beschrieben als in der ICD-10 und orientieren sich an den drei Symptomen der deskriptiven Definition, die ursprünglich auf Maslach/Jackson (1981/1984) zurückgeht und die diskursiv eine große Wirkkraft entfaltet hat (siehe Beleg 12 in Kap. 6.2.2.2) ${ }^{783}$ Durch die Aufnahme in dieses offiziell verabschiedete gültige Klassifikationssystem, das gesellschaftlich zum Beispiel im Krankenversicherungsrecht eingebunden ist, erhält die Definition in Kombination mit den Hauptdiagnosen deklarative Kraft, dadurch dass eine Person auf dieser Basis Leistungen des Gesundheitssystems und spezielle Therapieangebote in Anspruch nehmen kann. >Burnout « wird durch die Aufnahme in dieses System zudem dem Verantwortungsbereich des Gesundheitssystems zugewiesen und in der ICD-11 auch eindeutig als berufliches Phänomen beschrieben.

Obgleich >Burnout nur als Zusatzdiagnose gelistet ist, wird dennoch häufig fachextern und fachintern auf diesen ICD-Schlüssel verwiesen: in den WikipediaArtikelfassungen seit dem Jahr 2006 explizit mit einem Informationskasten im Kotext der deskriptiven Definition zu Beginn des Artikels (vgl. Kap. 6.2.3.2); in den klinischen Fachtexten und Medientexten, die Fachmeinungen zitieren oder Fach-

780 Vgl. den Eintrag zu >depressive Episode in der ICD-10. Online einsehbar unter: https://www. icd-code.de/icd/code/F32.1.html (zuletzt abgerufen am 16.12.2019, = DIMDI 2019c, im QV unter 8.1.3).

781 Vgl. den Eintrag zu >Neurasthenie code.de/icd/code/F48.0.html (zuletzt abgerufen am 16.12.2019, = DIMDI 2019d, im QV unter 8.1.3).

782 Vgl. den Informationsbeitrag dazu im Deutschen Ärzteblatt unter: https://www.aerzteblatt. de/nachrichten/95908/ICD-11-WHO-stellt-neuen-Diagnoseschluessel-vor (= me/hil/aerzteblatt. de (2018), im QV unter 8.1.4; zuletzt abgerufen am 16.12.2019).

783 Diese Definition ist einsehbar unter: https://www.who.int/mental_health/evidence/burnout/en/ (zuletzt abgerufen am 16.12.2019, = WHO (5/2019), im QV unter 8.1.2). 
leute interviewen, insbesondere seit den 2010er Jahren ${ }^{784}$, dabei häufig mit einem kritischen Impetus, der nun direkt im Anschluss durch einen eigenen Typ diskursiver Praxis des Definierens (Typ 5a) beschrieben wird, da es sich in diesem Fall um eine argumentativ-deskriptive Definitionsentfaltung handelt:

\section{Typ 5a: Eine sich argumentativ-deskriptiv entfaltende klassifizierende `Zustands`- Definition,}

- $\quad$ die eine (in anderen Texten des Diskurses scheinbar erfolgte) |Zuweisung des zu definierenden Ausdrucks/Begriffs/Sachverhalts zu einem nominalen Definiens-Ausdrucks höherer Abstraktion (z. B. (eigenständige) Krankheit/Erkrankung, Diagnose) mit Bezug auf ein geltendes taxonomisches Klassifikationssystem, das bestimmte Definitionskriterien voraussetzt, (explizit begründend) zurückweist|

- $\quad$ und |durch ein anderes Definiens-Nomen (z. B. Risikozustand) ersetzt|,

- das den |Ausdruck/Begriff/Sachverhalt unter neuen Voraussetzungen in das Klassifikationssystem einordnet|, //

- Typ 5b: /mit einer bestehenden Diagnose (z. B. Depression) gleichsetzt und damit unter diesem Schlüssel und Namen in das Klassifikationssystem einordnet|

- $\quad$ die teilweise im direkten Anschluss lein oder mehrere spezifizierenden Merkmal(e) des Begriffs/Sachverhalts charakterisiert|, (die sich diachron betrachtet diskursiv durchgesetzt haben), oder |Merkmalskombinationen benennt, durch die sich der Begriff/ Sachverhalt von sachverwandten Begriffen/Sachverhalten (wie z. B. der `Depression`, sreversibler Erschöpfungszustand`) unterscheidet oder diesen angleicht|;

- $\quad$ die |(implizit) auf durchschnittliche menschliche und gruppenspezifische Vergleichswerte und gesellschaftliche und kulturelle Erwartungsnormen verweist| und damit eine |Ein- und Abgrenzung des zu definierenden Begriffs/Sachverhalts jenseits der transportierten Normalitätsfolie stimuliert|;

- $\quad$ mit deklarativer Grundfunktion in fachlichen Übersichtsbeiträgen, medialen Problemdarstellungen und fachexternen Onlineportalen (z. B. Wikipedia).

\section{Typ 5c: Eine sich argumentativ entfaltende nach wissenschaftlichen Methoden ope- rationalisierte 'Zustands <-Definition,}

- die einzelne |charakteristische Attribute/Merkmalsausprägungen und Kombinationen, die dem zu definierenden Ausdruck/Begriff/Sachverhalt in anderen Texten operationalisierend zugewiesen wurden, anzweifelt/zurückweist oder erweitert| und durch andere/weitere charakteristische Attribute/Merkmalsausprägungen und Kombinationen ersetzt, dadurch dass neue |Items präsentiert und begründet werden,

784 Vgl. z. B. den „Kodier-Ratgeber zum Burnout-Syndrom im Dt. Ärzteblatt, 2011, 108 (17): A-976, = Deutsches Ärzteblatt 2011) oder die Bezüge auf die ICD-10 in den beiden Artikeln der Zeitschrift „Der Nervenarzt“ von Berger/Schneller/Maier (2012) und Berger/Gravert/Schneller et al. (2013); alle Belege im QV unter 8.1.4. In Medientexten findet man ebenfalls Bezüge auf die ICD, z. B. in der Süddeutschen Zeitung vom 22.10.2011: 24 (= Weber 2011) oder auf FAZ.NET am 02.03.2012 (= Belz 2012), im QV unter 8.1.6. 
die typische Verhalten- und Erlebensmerkmale von Personen in diesem Zustand operationalisieren|,

- die laufgrund empirisch-statistischer Reliabilitäts- und Validitätstests und einer methodisch kontrollierten Auswertung durch Fachleute postuliert, dass die operationalisierten Items den empirisch beobachteten Merkmalsausprägungen entsprechen| und |direktiv die einzelnen Versuchspersonen dazu auffordert, die Iteminhalte mit dem eigenen Erleben und Verhalten zu vergleichen und ihnen (graduell) zuzustimmen oder diese abzulehnen|,

- $\quad$ in einer fachlichen Originalarbeit oder einer fachlichen Handreichung zu einem Fragebogenmessinstrument, das überprüft wird oder in Abgrenzung zu anderen Messinstrumenten präsentiert wird ${ }^{785}$,

- $\quad$ mit repräsentativer Grundfunktion.

Typ 5 a wurde in Kapitel 6.2.2.2 an den Belegen 8a-2, 10 und $12^{786}$ und im Kapitel 6.2.3.1 in Abschnitten der Belege 10 und $11^{787}$ beschrieben. Die argumentative Definitionsentfaltung negiert ein im Diskurs etabliertes (terminologischtaxonomisches) Definiens-Nomen höherer Abstraktion (z. B. Diagnose, Krankheit, medical condition) und dessen Bedeutung im Rahmen einer bestehenden Taxonomie (z. B. der ICD-10 oder ICD-11) im Rahmen intertextueller Aushandlung und ersetzt es durch ein anderes Definiens-Nomen (z. B. Risikozustand, factor influencing health status). Sie negiert also die mit diesem Hyperonym vorgenommene Einordnungspraktik in bestehendes Wissen (Burnout ist keine Diagnose im Sinne der ICD-10) vor dem Hintergrund, dass >Burnout`im Gegensatz zu anderen

785 Eine Variation dieses Musters zeigt sich im Burnout-Diskurs, wenn der metaphorische Definiensausdruck Burnout abgelehnt wird, weil die Metapher und damit einhergehende Bedeutungsaspekte zu einer Konstruktannahme führen, die nicht der beobachteten und erlebten ,Wirklichkeit' entspricht. Dieses Muster erscheint im Diskurs jedoch erstaunlich selten. Vgl. das folgende Beispiel von Burisch (52014: 10, Unterstreichungen T.S.): „Bei genauerem Hinsehen erweist sich die Metapher »Burnout« übrigens als nicht allzu treffend, selbst dann, wenn man das Wort mit »Durchbrennen« übersetzte, was korrekter wäre. Durchbrennen können Sicherungen oder Stromleitungen, aber beides geschieht abrupt, und der Effekt ist sofortiger Stillstand, nicht die oft jahrelange Quälerei des Burnout. [...]. Was dagegen psychologisch-metaphorisch mit Burnout gemeint ist, ist eine lang dauernde zu hohe Energieabgabe für zu geringe Wirkung bei ungenügendem Energienachschub - etwa so, als wenn eine Autobatterie nicht mehr über die Lichtmaschine nachgeladen wird, dennoch aber Höchstleistungen abgeben soll." Weitere Belege siehe Kap. 4.2.2.

786 Weitere Belege aus dem fachlichen Korpus sind z. B. der HTA-Bericht von Korczak/Wastian/ Schneider (2012: 14, im QV unter 8.1.5), und in der Zeitschrift „Der Nervenarzt Rössler/Hengartner/Ajdacic-Gross (2013: 799) und Hamann/Parchmann/Mendel et al. (2013: 841); und in der Zeitschrift „Dt. med. Wochenschrift“ Kissling/Mendel/Förstl (2014: 2588); im QV unter 8.1.4.

787 Weitere Belege aus dem fachexternen Korpus Weiguny/Nienhaus (2014a, FAZ vom 19.4.2014), Kaulen (2010, FAZ vom 6.10.2010: 232, im QV unter 8.1.6); Eberle (2014: 28, GEOkompakt 08/2014); Apotheken Umschau (10/2011-A: 34, = Rotherbl 2011); beide im QV unter 8.1.7. 
Konzepten (wie >Depression`, >Neurasthenie`, >Angststörung`, >Tinnitus ) die definitorischen Aufnahmekriterien, um als >Diagnose in diesem System zu gelten, nicht erfüllt und deshalb systemintern anders eingeordnet werden muss. ${ }^{788}$ Auf der spezifizierenden Ebene werden im Anschluss an diese kritisch-substitutive Klassifizierungspraktik teilweise dennoch charakteristische und diskriminante Merkmale aufzählend oder deskriptiv entfaltet, die mitunter Kriterien benennen, die sich intertextuell schon im Diskurs durchgesetzt haben: So werden zum Beispiel die Merkmale (emotionale Erschöpfung, Zynismus, Leistungsminderung) der Definition, die auf Maslach/Jackson (1984) ursprünglich zurückgeht und am häufigsten einzeln oder hervorgehoben in den Fachlexika-, Hand- und Lehrbüchern und fachlichen Übersichtsartikeln präsentiert wird (siehe Typ 1 oben), als Spezifizierung mit dem neuen klassifizierenden Ausdruck/Konzept (>Risikozustand ) verbunden und zu den anderen klassifizierenden Ausdrücken/Konzepten (`Folgekrankheiten`, >Depression`, >Angststörung`, >Tinnitus`) in ein temporales und konditionales Beziehungsgefüge gestellt (vgl. dazu Beleg 10 in Kap. 6.2.2.2). Definitionen, die sich auf eine fachlich und gesellschaftlich etablierte Taxonomie beziehen, gehen mit einer deklarativen Grundfunktion einher, wobei durch die Zurückweisung des Hyperonyms Krankheit jedoch auch vermittelt wird, dass dem schriftlichen Vermerk der „Zusatzdiagnose“ gesellschaftlich-institutionell nicht dieselben Wirkmöglichkeiten zukommen wie dem einer „Hauptdiagnose“. Mit letzterer zusammen kann die Zusatzdiagnose jedoch zur Verschreibung spezifischer Therapiemaßnahmen, die auf sie abgestimmt sind, führen. ${ }^{789}$

Typ 5 b ähnelt dem Typ 5 a dadurch, dass er das Definiens-Nomen Krankheit für >BURNOUT< ebenfalls ablehnt, er unterscheidet sich aber von Typ 5a dadurch, dass er Burnout dann nicht als >Risikozustand ‘ für eine folgende psychische Erkrankung fasst, sondern mit einer anderen schon bestehenden psychischen

788 So zum Beispiel, wenn argumentiert wird, Burnout sei „keine Erkrankung, sondern ein aus Überlastungen resultierendes Phänomen eigener Art“ und keine medizinische Diagnose, da diese „klare Kriterien“ voraussetze, die dem Burnout-Konzept abgesprochen werden (vgl. Hillert (2012: B2, FAZ, 16.03.2012) und zu den „klaren Kriterien“ in fachsprachlichen Kontexten auch Kap. 4.2.2 bis 4.2.4).

789 Dies zeigt sich z. B. in folgendem Beleg, in dem die Bedingungen beschrieben werden, unter denen die Zusatzdiagnose verschlüsselt werden soll: „Die DGPPN empfiehlt deshalb, immer zuerst die jeweils zutreffende ICD-10-Diagnose (z. B. Depression oder Hypertonie) zu verschlüsseln. Wenn darüber hinaus angenommen wird, dass bei der Entstehung und Aufrechterhaltung dieser als Hauptdiagnose festgestellten Erkrankungen ein Erschöpfungszustand im Sinne eines Burnouts eine entscheidende Rolle gespielt hat, sollte zusätzlich die ICD-10-Anhangsziffer Z 73.0 codiert werden. Dadurch werde die krankheitsauslösende Überforderung eines Patienten gekennzeichnet und entsprechende spezifische Therapiemaßnahmen nahegelegt“ (Kissling/ Mendel/Förstl (2014: 2588, DMW, im QV unter 8.1.4, Unterstreichungen T.S.). 
Erkrankung (meist der >Depression`) gleichsetzt (vgl. Beleg 11 in Kap. 6.2.3.1 und SZ, 22.10.2011 (= Weber 2011: 24)).

Typ 5 c findet man vor allem in fachlichen Studien, die eine operationale Definition und deren Messinstrument überprüfen oder in Abgrenzung von bestehenden operationalen Definitionen und Messinstrumenten eine eigene Definition plus Messinstrument definieren (siehe Beleg 6b plus die Belege in Fußnote 508 in Kap. 6.2.2.2). Wie schon bei Typ 3a ausgeführt, geht es hier um ein möglichst adäquates Verhältnis zwischen fachsprachlich gefasster konzeptioneller Definition, sprachlich gefassten operationalisierten Fragebogenitems und den in der Realität beobachteten Merkmalen. Diese Definitionen werden vor einem repräsentativ-ontologischen Geltungshorizont formuliert.

Die dargelegten Typen 1-5 haben sich als dominierende Typen diskursiver Definierens-Praxis im Burnout-Diskurs aus den analysierten Belegen des fachlichen und fachexternen Diskursstrangs herauskristallisiert. Im Vergleich dieser Typen wurde deutlich, dass die Definitionsentfaltungsarten mit fach- und bereichskulturellen Praktiken und „kommunikativen und situativen Faktoren“ (Brinker/Cölfen/Pappert ( $\left.{ }^{8} 2014: 57\right)$ korrelieren. Das erkennt man unter anderem daran, dass bestimmte Definitionsentfaltungstypen bevorzugt in bestimmten fachlichen oder vermittlungssemantischen Umgebungen erscheinen: So findet man z. B. die narrative Definitionsentfaltung insbesondere in medialen Magazinberichten, die deskriptive Definitionsentfaltung des Typs 1 verstärkt in Fachlexika, Hand- und Lehrbüchern (medial erscheinen eher verkürzte Versionen und Mischformen) und die sich argumentativ entfaltende klassifizierende ZustandsDefinition des Typs 5a hauptsächlich in Fachtexten der klinischen Fächer und Pressetexten, die Expertinnen/Experten aus diesen Fächern zitieren. ${ }^{790}$

Es wurde im Vergleich des Weiteren sichtbar, dass eine Definition, die ursprünglich dem deskriptiven Typ 1b zuzurechnen ist und die dem aristotelischen Schema des „Genus proximum - differentia specifica“ formal ähnelt, aus transtextueller Perspektive die weiteste Verbreitung im Diskurs erfahren hat. Dies könnte damit zusammenhängen, dass diese Definitionsform sehr komprimiert und dadurch gut zitierbar ist und dass dieses prototypisch-klassische Definitionsmuster die Geltung der definitorischen Proposition erhöht.

Ein grundlegender Unterschied zwischen den Definitionstypen zeigt sich bezüglich der Ansprüche und Bedingungen, die die Geltung der Definition garantieren sollen:

790 Die klinischen Fächer sind im Gegensatz zu anderen psychologischen Fächern wie der Pädagogischen Psychologie, Sportpsychologie oder Arbeits- und Organisationspsychologie direkt in die Abrechnungspraktiken des Gesundheitssystems und damit in Klassifizierungspraktiken auf Basis der ICD eingebunden. 
Die Definitionstypen 1a-c, 2d, 3a-b und 5c werden mit dem Anspruch nach sachgemäßem Realitätsbezug als Realdefinitionen mit repräsentativer Funktion formuliert und vor diesem Hintergrund als wahre Tatsachen empirischer Beobachtung und/oder (psychologischer $\mathrm{u}$. medizinischer) Forschung behauptet (zur „realistischen Weltauffassung“, sprachphilosophischen Debatte, worauf Zeichen verweisen, und zu repräsentativen Aspekten des Definitionsakts siehe Kap. 2.1.1 und 4.3.2 dieser Arbeit). Diese Definitionen sollen intersubjektiv überprüfbar sein, sie behaupten jedoch nicht, allgemeingültig zu sein, in dem Sinne, dass sich die Mehrheit der Fachleute auf sie geeinigt hätte und dass diese Definition innerhalb eines Fach- und Praxisbereichs als alleinige Definition gelte. Diese repräsentativen Formen des Definierens, bei denen sich diejenige Definition vorläufig durchsetzen sollte, die sich in Bezug auf die beobachteten Verhältnisse oder durch ihre Verwendung in der Forschung zu bewähren scheint, ${ }^{791}$ passen zur Grundidee der „Logik der Falsifikation“ nach Popper (vgl. Küttner 1992: 80; Prechtl 21999: 175) und zum theoretisch unabschließbaren Sinnbildungsprozess nach Peirce (vgl. Kap. 2.2.2 dieser Arbeit; CP 8.148).

Die Definitionstypen 2a-c, 4a-b und 5a-b weisen die Definition als Teil eines offiziell verabschiedeten, international konsentierten Klassifikationssystems aus, das gesellschaftlich-institutionell eingebunden ist. Die jeweilige Definition ist gültig, wenn sie dort verzeichnet ist. Der „Streit um die Wahrheit“ des propositionalen Gehalts der definitorischen Behauptung, also z. B. der Streit um ihre Zuordnung zu einer höheren Kategorie oder um ihre inhaltliche Spezifizierung, gilt zumindest für die aktuell-geltende Version des Klassifikationssystems als abgeschlossen. Die deklarierenden Institutionen bringen den propositionalen Gehalt der jeweiligen Definition mit der Wirklichkeit/dem bestehenden Wissen dahingehend zur Deckung, dass jemand nur dann als >depressiv oder sausgebrannt gilt, wenn er die dort definierten Kriterien erfüllt (vgl. Searle 1980: 98; Saß/Saß-Houben 2005: 137ff.). Institutionelle Leistungen können dann auch nur je nach Status der Klassifizierung in diesem System in Anspruch genommen werden (zu deklarativen und repräsentativ-deklarativen Aspekten bei Definitionen vgl. Searle (1980: 99) und Kap. 4.3.2).

Diese Geltungsbereiche überkreuzen sich jedoch auch, und sie können sich dabei gegenseitig verstärken, wie zum Beispiel an den Typen 1a (deskriptive Lexikondefinition), 2a-c (narrativ-referierende Definitionen in Medientexten oder

791 Wobei damit nicht gesagt sein soll, dass nur weil ein Messinstrument oder eine Definition von verschiedenen Studien weiterverwendet wird, dies ihren Bewährungsgrad eindeutig belegt. Denn dies können auch diskursive Übernahmepraktiken sein, die die Probleme einer Definition/ eines Messinstruments im Rahmen der Studie nicht thematisieren. 
fachlichen Kasuistiken) und 3b (Definitionen über Symptomlisten/Fragebogen in fachexternen Medientexten) gezeigt wurde:

- Zum Beispiel erstens, wenn eine singulär erscheinende Definition in einem etabliert-anerkannten klinischen Fachwörterbuch von einigen Leser/innen als aktueller fachlicher Konsens bzw. deklarierte „endgültige Meinung“ interpretiert wird (vgl. Peirce, CP 8.148), auf deren Basis institutionell Entscheidungen getroffen werden können (vgl. Kap. 4.3.2); Wenn auf diese repräsentative Definition zudem häufig intertextuell in einem Diskurs Bezug genommen wird und sie gegenüber anderen Definitionsvarianten hervorgehoben erscheint und eine wirkmächtige Position im Diskurs erhält, dann erhöht sich die Wahrscheinlichkeit, dass sich eine Definition für ein Klassifizierungssystem mit deklarativer Geltungsmacht an dieser repräsentativen Definition orientiert (vgl. dazu die obigen Ausführungen, dass die Symptomtrias der Definition, die auf Maslach/Jackson 1981/1984 zurückgeht, in modifizierter Form in die ICD-11 aufgenommen wird, die ab 2022 die ICD-10 ablöst);

- Zweitens, wenn auf eine Erzählung, die den Anspruch erhebt, die Symptome 'wirklichkeitsgetreu' wiederzugeben, der deklarative Akt des Diagnostizierens folgt, und zwar von einer Person, die in das Klassifikationssystem institutionell eingebunden ist;

- Drittens, wenn eine Liste oder ein Fragebogen im fachexternen Diskursstrang die typischen Symptome für >Burnout` einerseits mit repräsentativer Funktion darstellt, und andererseits in Bezug auf diese Symptome eine Warnung an die Leser/innen ausspricht: Wenn sich die Leser/innen mit den Symptomen identifizieren, sollen sie Fachpersonen des klinischen Bereichs konsultieren. Von diesen können sie dann möglicherweise auf der Basis einer deklarativen Definition krankgeschrieben werden.

In diesen drei Fällen werden die repräsentativen und deklarativen Geltungsansprüche zusammengeführt und der repräsentative Definitionsakt erhält zusätzlich deklarative Kraft.

Es gibt aber auch Fälle, in denen repräsentative und deklarative Geltungsansprüche innerhalb von Definitionstypen in Konflikt zueinander geraten können bzw. in denen der Zusammenfall von Geltungsansprüchen (wie im gerade beschriebenen zweiten narrativen Beispiel) im Diskurs kritisiert wird. Dies ist der Fall im Definitionstyp 5a. Das Metakonzept >Krankheit` wird als deklaratives Klassifizierungskonzept zurückgewiesen. Doch wie kommt es zu solchen divergierenden Geltungsansprüchen im Kontext des Definierens? Dieser Frage wird im folgenden Kapitel vor dem Hintergrund der Analysekonzepte der `Unifizierung، und >Agonalität ‘ weiter nachgegangen. 
Betrachtet man abschließend, in welchen Punkten sich alle Definitionstypen aus transtextueller und diachroner Perspektive ähneln, so zeigen sich zwei Faktoren als äußerst stabil: In jedem Definitionstyp findet man Praktiken, die die definitorischen Propositionen verallgemeinern und entweder auf alle Menschen oder ähnliche Personengruppen generisch referierend beziehen. Des Weiteren werden über die verschiedenen Definitionstypen hinweg (implizit) durchschnittliche menschliche und gruppenspezifische Vergleichswerte und gesellschaftlich-kulturelle Erwartungsnormen zur Ein- und Abgrenzung des zu definierenden Begriffs/Sachverhalts aufgerufen (vgl. dazu die Analysen in Kap. 6.2.4.1). Von diesen Wissensvoraussetzungen scheint eine unifizierende Wirkung auszugehen, da sie in verwandter Weise in fachlichen und fachexternen Texten aufgerufen werden, obgleich Unterschiede in den Wissensgrundlagen auf Experten- und Laienseite aufgezeigt werden können und die Formulierungen in unterschiedlichen Abstraktionsgraden erscheinen.

Bisher wurden die sprachlichen Mittel und Praktiken des Definierens im Hinblick auf ihr unifizierendes Potenzial beschrieben. Im folgenden Kapitel wird der Frage nachgegangen, welchen Einfluss sprachliche Mittel und Praktiken der Agonalität auf sprachliche Mittel und Praktiken des Definierens haben können und wie sich Dimensionen der Unifizierung und Agonalität in den Definitionstypen im Burnout-Diskurs gegenseitig bedingen. Es soll ferner um die Frage gehen, welche Analysewege das Modell einer diskursiven Praxis des Definierens eröffnet, um unifizierende und agonale Zentren in einem Diskurs aufzuspüren.

\subsection{Modell der diskursiven Praxis des Definierens im Spannungsfeld von sUnifizierung` und `Agonalität`}

In Kapitel 6.2.1 wurden die Schritte der in dieser Arbeit durchgeführten Analyse mit dem Konzept der >Unifizierung` umschrieben. Dieses Analysekonzept konzentriert sich auf sprachliche Mittel und diskursive Praktiken, durch welche die Polyphonie unterschiedlicher diskursiver Stimmen bzw. das ,Vorhandensein verschiedener konkurrierender Positionen zu einem Thema“ (vgl. Mattfeldt 2018: 54) im Diskurs reduziert wird und einige Wissensbestände in Form von Definitionen „diskursiv weiter bearbeitet werden, andere jedoch keine prominente Erwähnung finden“ (Felder 2013: 15). Diesen sprachlichen Zeichenkombinationen und Praktiken kommen vereinheitlichende, verdichtende und verknappende Wirkungen zu, wodurch sie den Prozess der diskursiven Hausbildung einer 'allgemeingülti- 
gen' Definition ${ }^{792}$ unterstützen. Der Aspekt der Allgemeingültigkeit bezieht sich auf das regulative Ideal, dass „eine endgültige Meinung“ im eigentlich unabschließbaren Sinnbildungsprozess zu einem Thema möglich sei (vgl. Peirce, CP 8.148 und Kap. 2.2.2) und dass eine soziale Gruppe per definitorischer Einigung den „Streit über die Wahrheit einer Behauptung zu einem Ende kommen [lassen] kann“ (Searle 1980: 99; vgl. auch Kap. 4.3.2 dieser Arbeit).

In den Unterkapiteln der Kapitel 6.2.2 und 6.2.3 $3^{793}$ wurden beispielsweise sprachliche Mittel herausgearbeitet, die innerhalb oder im direkten Kontext der punktuellen Definitionsakte explizit auf eine Konsensfindung im Diskurs referieren (das ist derzeit fachlicher Konsens), Einzelfallbeispiele verallgemeinern (jeder neunte Deutsche ist betroffen) oder einzelne Aspekte des Begriffs >Burnout und einzelne Definitionen gewichten (das wichtigste Erkennungszeichen; ein deutliches Symptom für Burnout; ...) und den Begriff extensional durch die generische Referenz auf Gruppen, die (besonders) ,betroffen' sind (Arbeitnehmer, insbesondere in Sozial- und Pflegeberufen) festlegen und eingrenzen. In den Kapiteln 6.2.4.1-6.2.4.3 wurde der Analyseblick geweitet und in diesem Zusammenhang gefragt, welche wiederholt in der Fläche der Diskursstränge auftretenden Zeichenkombinationen Effekte stimulieren können, die eine Verknappung, verfestigende Gewichtung und (bereichsspezifische) Geltungsvalidierung bestimmter Definitionsvarianten und Zuordnungspraktiken bewirken können. Es wurde unter anderem auch im letzten Kapitel bei der Formulierung der Typen diskursiver Definierenspraxis gezeigt, dass bestimmte strukturell-funktionale Kombinationen definitorischer Praktiken (z. B. Typ 1) eine später erfolgende intertextuelle Wiederaufnahme der Definition begünstigen können, wodurch diese Variante nach und nach eine dominante Diskursposition erhalten kann. Des Weiteren wurde im letzten Kapitel an den verschiedenen Typen erläutert, unter welchen Bedingungen Definitionen im Bereich psychischer Gesundheit und Krankheit eine nicht nur repräsentative, sondern deklarative und dadurch gesellschaftlich verbindliche Kraft entfalten können.

Trotz dieses unifizierenden Analysefokus wurde von Beginn an betont, dass im Burnout-Diskurs auch semantische Dimensionen der Agonalität (vgl. Felder 2012; 2013; 2015; Mattfeldt 2018) und semantische Kämpfe (vgl. Felder 2006b; Busch 2006) eine Rolle spielen. Deren Einfluss auf das Analysemodell und ihre

792 Dieser diskursive Prozess wurde basierend auf der Arbeitsdefinition einer diskursiven Praxis des Definierens in dieser Untersuchung als überindividueller, transtextueller chronologischer Prozess konzeptualisiert (siehe Kap. 4.3.4 und 7.1).

793 Siehe im Überblick die Tabellen 1, 2.2, 4 und 5.2 in den Zusammenfassungskapiteln 6.2.2.4 und 6.2.3.3. 
Verbindung zu sprachlichen Mitteln der Unifizierung im Kontext diskursiver Praktiken des Definierens soll im Folgenden erörtert werden.

Einen aktuellen Überblick zur Agonalitätsforschung gibt Mattfeldt (2018: 52ff.). Sie verortet die linguistische Agonalitätsforschung mit Rückgriff auf Warnke (2009), Felder (2015) und Niehr (2014) in der linguistischen Diskursanalyse. „In der Linguistik liegt der Fokus auf Wissensbeständen in einer Gesellschaft, die diskursiv ausgehandelt werden“ (Mattfeldt 2018: 53). Felder zufolge zeigen sich diese Aushandlungsprozesse um Wissensbestände in einer Gesellschaft, wenn „zwei oder mehr Konzepte in einem Diskurs konfligieren und für einen Diskurs als charakteristisch gelten“ (Felder 2013: 21). Diese Streitpunkte lassen sich nach Felder in „agonalen Zentren“ kristallisieren, die er folgendermaßen definiert:

Unter agonalen Zentren verstehe ich einen sich in Sprachspielen manifestierenden Wettkampf um strittige Akzeptanz von Ereignisdeutungen, Handlungsoptionen, Geltungsansprüchen, Orientierungswissen und Werten in Gesellschaften. Im Fokus der Aufmerksamkeit stehen kompetitive Sprachspiele zwischen verschiedenen gesellschaftlichen Diskursakteuren.

(Felder 2013: 21)

Mattfeldt verwendet einen weiter gefassten Agonalitätsbegriff, der Agonalität „als Polarität und Opposition an sich versteht, die sich sprachlich manifestiert“ und die nicht zwingend an menschliche Akteure "gekoppelt sein“ muss (Mattfeldt 2018: 55f.). Denn:

In einem übertragenen Sinne werden auch Ideen zueinander in Rivalität gesetzt, ebenso Objekte und Anschauungen; diese werden zwar von Menschen in Konkurrenz gesetzt, aber diese müssen nicht explizit genannt werden und können in den Hintergrund treten.

(ebd.: 55)

Sie definiert Agonalität daran anschließend für Ihre Untersuchung wie folgt:

Agonalität geht auf Wettkämpfe in der Antike zurück und bezeichnet eine breit verstandene kompetitive Opposition oder Polarität, die nicht zwingend an menschliche Akteure gebunden ist. Agonalität manifestiert sich auf der sprachlichen Oberfläche (weit verstanden und damit auch visuelle Inhalte einschließend).

(ebd.: 56)

Diese weite begriffliche Fassung des Analysekonzepts der `Agonalität` lässt sich gut mit dem in dieser Arbeit formulierten Analysekonzept der `Unifizierung ‘ verbinden. Denn letzteres nimmt mit Bezug auf den Arbeitsbegriff einer diskursiven Praxis des Definierens insbesondere auch nicht intendierte, unwillkürliche, überindividuelle Praktiken in den Blick, die über das Wechselspiel von intra- und transtextueller Analyse erfasst werden können. 
Dass es einen „Wettkampf“ um die Geltung und Akzeptanz von Sachverhaltsdeutungen und -festsetzungen im Burnout-Diskurses gibt, zeigt sich explizit an dem Umstand, dass die Erwartungen an die Tätigkeit des Definierens aus Textstellen des fachlichen und fachexternen Diskursstrangs extrahiert wurden, die eine uneinheitliche, nicht eindeutige, zu wenig trennscharfe und ungenaue Verwendung des Begriffs >Burnout « und eine fehlende allgemeingültige bzw. international konsentierte Definition konstatieren (siehe Kap. 1.2; 4.2.2 und 4.2.4). Diese kritischen metasprachlichen Äußerungen von Diskursakteuren insbesondere aus dem klinischen Bereich, die für sich genommen „Sprachhandlungsmuster“ des semantischen Kampfs darstellen, ${ }^{794}$ stehen allerdings dem konvergenten Leseeindruck, der sich in Bezug auf die begriffliche Fassung bzw. Definition des Phänomens/Begriffs >BuRnout aus transtextueller, diachroner Perspektive einstellt, entgegen. ${ }^{795}$ Des Weiteren transportieren Sprachhandlungsmuster des semantischen Kampfs, die den vorliegenden Definitionen eine „uneindeutige Begriffsbestimmung“ oder „das Fehlen allgemeiner Verbindlichkeit“ vorwerfen (vgl. Kap. 4.2.2) zugleich Ideale der Eindeutigkeit und allgemeinen Verbindlichkeit, die, wenn sie häufig in Texten des Diskurses vorgetragen werden, eine legitimierenden Grundlage für unifizierende Praktiken bieten. Diese Beobachtungen legen den Schluss nahe, dass agonale und unifizierenden Dimensionen im Burnout-Diskurs im Kontext der Praxis des Definierens in besonderer Weise wechselseitig aufeinander bezogen sind.

Einen ähnlichen Bezug zwischen agonalen und konsensualen Dimensionen stellt Mattfeldt (2018: 99) in ihrer diskursvergleichenden Studie zur Agonalität im Deutschen und Englischen her, wenn sie das „Beenden des agonalen Zustands“ als eine semantische Dimension der Agonalität ausweist. Mattfeldt beschreibt

794 Busch beschreibt „semantische Kämpfe in der Medizin“ als „Sprachhandlungsmuster (Zweck-Mittel-Konfigurationen, die in allen Kampfesformen auffindbar sein können)“(Busch 2006: 65f.). Besonders die Sprachhandlungsmuster „Wissenschaftlichkeit absprechen“ oder „methodologische Fehler vorwerfen “ ähneln den Äußerungen im fachlichen, aber auch öffentlich-medialen Burnout-Diskurs, die Kritik an der Güte der jeweiligen Definition des ,BURNOUT-SYNDROMS ‘ üben (vgl. Kap. 4.2.2). Man könnte diese damit auch als Sprachhandlungsmuster des semantischen Kampfs im Kontext von Definitionen des Burnout-Syndroms kategorisieren (z. B. das Sprachhandlungsmuster: „Uneindeutige Begriffsbestimmung vorwerfen“ oder allgemein „methodologische Fehler beim (wissenschaftlichen) Definieren vorwerfen“.

795 Die Arbeit schließt damit an die Programmatik Felders für linguistische Diskursanalysen an: „Diskursanalysen sollten die besonders umstrittenen als auch die vermeintlich so konsensual und unwidersprochen vermittelten Konzepte transparent machen - und zwar so, wie sie in der Öffentlichkeit präsentiert werden, und nicht so, wie bestimmte Diskursakteure behaupten, dass die Gegenstände in den Medien präsentiert würden. Das ist ein ganz entscheidender Unterschied.“ (Felder 2013: 20). 
Sprachgebrauchsformen im Englischen ${ }^{796}$, mit denen versucht werde, Konsens herzustellen. Die „versöhnliche Perspektivierung“ impliziere jedoch, „dass die Situation nicht immer konsensual war“ (ebd.). Im Burnout-Diskurs erscheint dieses Wechselverhältnis spiegelbildlich: Die Kritik im Burnout-Diskurs an der Güte der Definition und begrifflichen Fassung von Burnout, vor allem seit den 2010er Jahren (und demnach knapp 35 Jahre nach Beginn des Diskurses, siehe Kap. 6.1), scheint ein Indiz dafür zu sein, dass sich verfestigte Perspektiven auf das Phänomen/Konzept im Diskurs etabliert haben, die von den Kritikern nicht geteilt werden bzw. als nicht fachkonform angesehen werden. Es geht im Wechselverhältnis agonaler und unifizierender Dimensionen im Kontext diskursiver Praktiken des Definierens also um die Fragen:

- Welche Sprachgebrauchsformen und Praktiken bewirken diese unifizierten Interpretationsperspektiven auf ein Phänomen in einem Diskurs?

- Mit welchen sprachlichen Mitteln können diese sprachlich verfestigten, unifizierten Perspektiven auf ein Phänomen wieder aufgebrochen werden?

Diese Fragen sollen im Folgenden am Beispiel der argumentativen Definitionsentfaltung des Typs 5a erörtert werden. Denn in dieser argumentativen Definitionsentfaltung findet man mustergültig die Praktik des |Aufbrechens unifizierter Interpretationsperspektiven|. In diesem Typ wird der zu definierende Ausdruck/Begriff/ Sachverhalt dadurch entfaltet, dass zu Beginn der Definition ein mutmaßlich im Diskurs etablierter, den Definiendum zugeordneter nominaler Ausdruck zurückgewiesen und durch einen neuen Ausdruck ersetzt wird. Zur Veranschaulichung sei exemplarisch nochmals der folgende Auszug aus einem fachlichen Übersichtsbeitrag zum Thema „Burnout“ aus dem Jahr 2014 zitiert (Unterstreichung T.S.):

\section{Kissling/Mendel/Förstl (2014: 2588) in der Zeitschrift „Dt. med. Wochenschrift“ (Unterstreichungen T.S., im QV unter 8.1.4):}

Laut der Deutschen Gesellschaft für Psychiatrie und Psychotherapie, Psychosomatik und
Nervenheilkunde (DGPPN) ist Burnout keine nach ICD-10 diagnostizierbare Krankheit,
sondern ein Erschöpfungs- und Überforderungssyndrom, das einen Risikozustand für eine
spätere - nach ICD-10 diagnostizierbare - psychiatrische oder körperliche Krankheit dar-
stellt (wie z. B. eine Depression oder eine Hypertonie) [3]. Diese Einschätzung wird auch

796 Mattfeldts Beispiele zu dieser semantischen Dimension der Agonalität stammen aus „politischen Diskursen“, in denen Themen wie „Fracking“ und „Hurricans“ verhandelt werden und in denen es „vor allem um Konflikte zwischen Politikern/und oder Parteien“ geht: „Since the latter's [Chris Huhne's, Anm. AM] departure, his language has been tempered. 'George [Osbrne, Anm. AM] has drawn a line under the antagonistic stuff now Chris has gone,' says one Tory minister“ (Mattfeldt 2018: 99). 
von der Mehrheit der Psychiater und Psychotherapeuten geteilt [6]. Die DGPPN empfiehlt deshalb, immer zuerst die jeweils zutreffende ICD-10-Diagnose (z.B. Depression oder Hypertonie) zu verschlüsseln. Wenn darüber hinaus angenommen wird, dass bei der Entstehung und Aufrechterhaltung dieser als Hauptdiagnose festgestellten Erkrankungen ein Erschöpfungszustand im Sinne eines Burnouts eine entscheidende Rolle gespielt hat, sollte zusätzlich die ICD-10-Anhangsziffer Z 73.0 codiert werden. Dadurch werde die krankheitsauslösende Überforderung eines Patienten gekennzeichnet und entsprechende spezifische Therapiemaßnahmen nahegelegt.

\section{$[\ldots]$}

Die oben dargestellten komplizierten Abgrenzungen können allerdings nicht darüber hinwegtäuschen, dass vieles davon empirisch noch wenig untermauert ist, [...].

Die Definition entfaltet sich über die explizite Zurückweisung einer Bezeichnungskette (keine nach ICD-10 diagnostizierbare Krankheit) und durch die anschließende Angabe einer Bezeichnungsalternative, die nach Felder (2006) und Fellbaum/Felder (2013: 180) in einem Diskurs als Indikator für semantische Kämpfe und Agonalität gedeutet werden kann. Mattfeldt spricht in diesem Zusammenhang von der agonalen Dimension der „lexikalischen Gegenüberstellung“ (Mattfeldt (2018: 92). Das agonale Zentrum, das im Burnout-Diskurs durch diese Benennungssubstitution ab den 2010er Jahren wiederholt in verschiedenen Texten verbalisiert wird, lässt sich folgendermaßen beschreibungssprachlich fassen: ১Burnout ist ein Zustand, der typische bzw. erforderliche Merkmale einer (psychischen) Krankheit/Diagnose nach Kriterien fachlich anerkannter deklarierter Klassifikationssysteme aufweist ‘ versus >Burnout ist ein Zustand, der typische bzw. erforderliche Merkmale einer (psychischen) Krankheit/Diagnose nach Kriterien fachlich anerkannter deklarierter Klassifikationssysteme NICHT aufweistr.

Dieses Beispiel ist unter drei Aspekten interessant: Erstens zeigen sich in den einander gegenübergestellten Benennungen divergierende Sichtweisen auf den Sachverhalt/Begriff >BURNout‘. Zweitens scheint die zurückgewiesene Bezeichnung Krankheit schon eine dominante Wirkkraft im Diskurs entfaltet zu haben. Denn eine diskursiv marginale Bezeichnungsalternative würde nicht in dieser deutlichen Form zurückgewiesen werden. Drittens wird von den Autoren, die aus dem medizinischen Bereich stammen, durch die Zurückweisung der Bezeichnung Krankheit auch die alleinige Verantwortlichkeit ihrer Wissensdomäne für das Phänomen BuRNout relativiert. Interessant ist in diesem Zusammenhang, welche sprachlichen Mittel und Praktiken diese dominante Positionierung und Verantwortlichkeitszuschreibung, gegen die sich die Abgrenzung richtet, diskursiv bewirkt haben. Dies sei im Folgenden mit jeweils passenden Verweisen auf die in Kapitel 6 durchgeführten Analysen nochmals rekapituliert: 
Zunächst finden sich im Burnout-Diskurs zahlreiche sprachliche Stimuli in fachlichen und fachexternen Texten, die Burnout im Verantwortungsbereich der Medizin und Psychologie verorten. In Kap. 6.2.4.1 wurde für den fachlichen und fachexternen Diskursstrang z. B. ausgewertet, aus welchen Fachbereichen die Autorinnen und Autoren von Texten stammen, die über das Thema „Burnout“ schreiben oder die in Medientexten zu diesem Thema interviewt werden. Die Beleglage hat ergeben, dass die Erforschung von und der Umgang mit Burnout sowohl von fachlicher als auch von fachexternen Seite primär den Fächern Wirtschafts-, Organisations- und Arbeitspsychologie, Medizin, Psychiatrie, Psychologie und Schnittstellenfächern wie z. B. der Arbeitsmedizin, medizinischen Psychologie zukommt bzw. zugewiesen wird, gefolgt von Fächern wie der Arbeitssoziologie, Versorgungsforschung, Sportpsychologie oder den Gesundheitswissenschaften. Diese Befunde zeigen zusammen mit den Ergebnissen der Keyword-Auswertung in den Fachzeitschriftenartikeln, dass >Burnout ‘ stabil in der Mitte zwischen den Forschungsperspektiven der Patho- und Salutogenese und damit zwischen den Praxisbereichen der einerseits klinischen und andererseits präventiven Medizin, Psychologie und Therapie sowie im Bereich des Forschungsthemas „Krankheit/ Gesundheit von Arbeitnehmer/innen“ verortet wird (siehe Kap. 6.2.4.1).

Doch wie ist es dazu gekommen, dass das Konzept `Krankheit ‘ - trotz dieser Verortung zwischen medizinischen und gesundheitspräventiven Verantwortungsbereichen - dem Begriff >BURNOUTく scheinbar fest zugeordnet wird. Handelt es sich dabei ,nur' um eine Gegenüberstellung von Experten- und Laienwissen (Busch 2006: 52), wie folgendes SPIEGEL-Zitat aus dem Jahr 2012 suggeriert?

Und all das, obwohl Burnout im medizinischen Sinne gar keine Diagnose ist, sondern allenfalls ein anderes Wort für Depression. Der Volksmund aber interpretiert das völlig anders und versteht darunter eine scheinbar neue und eigenständige Krankheit, charakterisiert durch pathologische Erschöpfung im Beruf.

(= Blech 2012: 124f., DER SPIEGEL 6/2012, Unterstreichung T.S., im QV unter 8.1.6)

Die vergleichenden Analysen von sprachlichen Mitteln und Praktiken der Abgrenzung/Polarisierung und Geltungsvalidierung im fachlichen und fachexternen Diskursstrang (siehe Kap. 6.2.2.-6.2.4) und der Bezug auf Attribute, die sowohl fachlichen als auch alltagssemantischen Krankheitsbegriffen zukommen, offenbaren noch weitere Erklärungsansätze. Denn medizinische und gesellschaftlich-öffentliche Krankheitskonzepte sind zum einen, wie in Kapitel 3.2 beschrieben wurde, aufeinander bezogen ${ }^{797}$, zum anderen wird `Burnout durch

797 Der Rechtsbegriff knüpft z.B. an medizinische und alltagssemantische Krankheits- und Störungskonzepte an, wie der Rechtswissenschaftler Huster in einem Beitrag zur „Bedeutung 
|Praktiken der Abgrenzung und Polarisierung| in den analysierten Definitionsabschnitten insgesamt stärker dem 'unerwünschten', 'anormalen' >Krankheitspok als dem gewissermaßen unmarkierten, 'normalen', 'erwünschten' >Gesundheitspolı zugeordnet:

- Das zeigt sich erstens dadurch, dass `Burnout` in einigen fachlichen und in vielen fachexternen Texten (dabei ganz besonders in den narrativen Definitionsentfaltungen, siehe folgend die Erläuterungen in den Fußnoten) als Körper- und Geisteszustand konstituiert wird, dem Attribute zugesprochen werden, die sowohl in medizinischen, juristischen als auch alltäglichen Krankheits- und Störungskonzepten eine wichtige Rolle spielen (vgl. dazu auch Kap. 3.2., 6.2.2.-6.2.3 und 6.2.4.2):

- z. B. der Aspekt der 'Behandlungs- bzw. Hilfsbedürftigkeit einer Person, die diese Merkmale aufweist', ${ }^{798}$

- die Komponente der 'Arbeitsunfähigkeit bzw. Funktions- und Leistungseinschränkung durch die entstandenen Merkmale/Symptome,799

des Krankheitsbegriffs für das Krankenversicherungsrecht“ beschreibt: Der Gesetzgeber habe nie versucht, den Krankheitsbegriff gesetzlich zu definieren, „trotz seiner zentralen Bedeutung“. Denn „ein derartiger Versuch“ wäre „der Fähigkeit des Begriffs, neue medizinische Erkenntnisse und Verfahren in die Versorgung zu integrieren, [...] auch kaum förderlich gewesen“ (Huster 2017: 42). Die Sozialrechtsprechung hingegen arbeite „mit einem zweigliedrigen Begriff, nach dem eine Krankheit ein regelwidriger Körper- oder Geisteszustand ist, der behandlungsfähig und -bedürftig ist (und/oder zu Arbeitsunfähigkeit führt)“ (ebd.: 43). Huster resümiert, dass der Krankheitsbegriff im Krankenversicherungsrecht „funktional und normativ“ sei. Und zur näheren Bestimmung dieses Krankheitsbegriffes schreibt er: „Er ist sicherlich nicht leer, weil es ein robustes medizinisches und Alltagsverständnis von Krankheit gibt, an das der Rechtsbegriff in vielen Fällen anknüpfen kann“ (ebd.: 50). Es wird also explizit eine Verbindung zwischen fachsemantischen und alltagssemantischen Krankheitsbegriffen konstatiert (vgl. dazu auch Kap. 3.2). Wenn somatische Symptome wie SCHLAFPROBLEME oder HÄUfIGE ERKÄLTUNGSKRANKHEITEN aufgelistet werden, dann kommt es auch zu somatischen Funktionseinschränkungen. Das Attribut der 'Arbeitsunfähigkeit' wird allerdings vorwiegend in narrativen Definitionsentfaltungen im fachexternen Diskursstrang konstituiert.

798 Das Attribut 'Behandlungs- bzw. Hilfsbedürftigkeit' wird sowohl in einem Fachlexikon angedeutet, wenn „im fortgeschrittenen Stadium Psychotherapie“ empfohlen wird (siehe z. B. Beleg 4 in Kap. 6.2.2.1), als auch in narrativen Erzählungen, bei denen eine Person am Ende erkennt, dass sie zum Arzt/Therapeuten gehen muss (siehe z. B. Beleg 5 in Kap. 6.2.3.1), oder in einem medialen Vermittlungstext, wenn die „chronische Erschöpfungsspirale“ des „Burnout“ explizit von einer 'normalen' Erschöpfung dadurch unterschieden wird, dass die „Betroffenen [...] Hilfe von außen“ brauchen (SZ 2008b, im QV unter 8.1.6). Das Attribut der 'Behandlungsbedürftigkeit' wird in fachexternen Narrationen allerdings häufiger aufgerufen als in deskriptiven Definierensformen (fachintern und fachextern).

799 Das Attribut 'Leitungseinschränkung’ wird durch das Symptom „(Gefühl) reduzierter Leistungsfähigkeit“ in den meisten fachlichen und fachexternen Definitionen konstituiert. In vielen 
- $\quad$ oder auch die weitgehende 'Unverschuldetheit bei der Entstehung der als nicht normal bzw. ungesund klassifizierten Merkmale’ bzw. die 'Eigendynamik' der sich zeigenden/ diagnostizierten Merkmale. ${ }^{800}$

- Zweitens wird die Zuordnung zum `Krankheitspolı dadurch verstärkt, dass definitionsübergreifend Aspekte des Konzepts >Burnout` (inferierten) gesellschaftlich-(fach)kulturellen Vorstellungen von 'durchschnittlichen', 'normalen', 'gesunden' und 'erwünschten' Erlebens- und Verhaltensweisen ,agonal“ gegenübergestellt werden (vgl. insbesondere die Kap. 6.2.2.1, 6.2.2.2, 6.2.3.1 und 6.2.4.1). Die sprachlichen Mittel, die diese Gegenüberstellung zwischen Vergleichszuständen/-merkmalen und Burnoutzuständen/-merkmalen indizieren, erstrecken sich vom Einsatz adversativer, substitutiver und konzessiver Konnektoren, ${ }^{801}$ über sprachliche Mittel der Negation, ${ }^{802}$ Gradierung und

fachlichen Texten wird aber eher vom Gefühl reduzierter Leistungsfähigkeit gesprochen - in fachexternen narrativen Definitionen wird daraus häufig eine Leistungsunfähigkeit der Beispielperson, die bis zur Arbeitsunfähigkeit gesteigert wird. Fachliche Definitionen sind in diesem Punkt oft vage.

800 Die Attribute der 'Unverschuldetheit' und 'Eigendynamik' hängen zusammen und können beispielsweise durch die Analyse semantischer Rollen (Primus 2012) in den unterschiedlichen Definitionstypen festgemacht werden (siehe die Analyse in Kap. 6.2.4.2): Die Personen erscheinen in den verschiedenen Definitionen überwiegend als Leidtragende, Erlebende der Burnout-Symptome, eingeschränkt Handelnde oder als vormalige Besitzer/innen von Handlungsmöglichkeiten und Fähigkeiten, die sie verloren haben (vgl. dazu die Analysen zu semantischen Rollen in Kap. 6.2.4.2). Sie haben diese Zustände nicht willentlich herbeigeführt, die Symptome treten in den Texten häufig ähnlich wie Wetterzeichen 'eigendynamisch' in Subjektposition und personifiziert auf (vgl. Peirce, CP 8.185; Kap. 3.1), was sich z. B. in folgenden Formulierungen zeigt: ,immer wieder auftretendes Herzrasen“ (Kissling/Mendel/Förstl 2014: 2587, DMW, im QV unter 8.1.4); ,es kann jeden treffen“ SPIEGEL ONLINE (2012b, im QV unter 8.1.6); „Zunehmend wandelte sich die Freude am Job in Abscheu“ (Falkai 2016: 20, weitere Belege in Kap. 6.2.4.2). Zur Konstituierung von 'Unverschuldetheit' und 'Kontrollierbarkeit' in der BurnoutRatgeberliteratur siehe auch Schnedermann (i. Ersch.).

801 Beispielbelege: „Der erste Pinselstrich fiel ihr schon immer schwer. Seit ein paar Tagen war es aber irgendwie anders.“ (AU 1997: 20, Unterstreichung T.S., im QV unter 8.1.7); „Ferien lassen nicht neue Kräfte schöpfen, sondern den Zwang zum Widerantreten der Arbeit nur düsterer scheinen.“ (Schmidbauer 1982: 165, Unterstreichung T.S., im QV unter 8.1.5); „Ich war nur noch müde. Einmal hatte ich sogar in meinem Auto auf einem Parkplatz übernachtet. Die Müdigkeit hatte mich so plötzlich überfallen, dass ich nicht mehr weiterfahren konnte, obwohl es nur noch zehn Kilometer bis zu meiner Wohnung gewesen wären. “(Abé 2012, SPIEGEL ONLINE, 08.08.2012, im QV unter 8.1.6, Unterstreichung T.S.)

802 Beispielbelege (Unterstreichungen T.S.): „Unvermögen, sich zu entspannen“ (Geyerhofer/ Unterholzer 2009, PiD, im QV unter 8.1.4); „gefühllose, gleichgültige Einstellung“ (Bauer/Häfner/Kächele et al. 2003, PPmP); „, führen kurze Erholungsphasen nicht zu einer Rückbildung“ (Berger/Falkai/Maier 2012, Dt. Ärzteblatt, im QV unter 8.1.4); weitere Beispiele in den Kapiteln 6.2.2.1; 6.2.2.2; 6.2.3.1 und 6.2.3.2. 
Quantifizierung, ${ }^{803}$ weiter über nominale oder adjektivische Terme, die antonyme und komplementäre Gegensatzpole evozieren, ${ }^{804}$ bis hin zu Zeitangaben und Tempusmarkierungen, die einen Kontrast zwischen Ausgangs- und Endzustand herstellen ${ }^{805}$. Bei der Konstituierung dieser Gegensätze kommen auch Aspekte zum Tragen, die Mattfeldt (2018) als semantische Dimensionen der Agonalität herausgearbeitet hat. ${ }^{806}$ Man sieht daran, dass im Bereich psychischer Gesundheit und Krankheit mit sprachlichen Mitteln operiert wird, die eine agonale Grundstruktur konstituieren. In Wissensbereichen, die sehr binär konstituiert sind, können sprachliche Mittel der Agonalität demnach unifizierende Wirkung entfalten.

- Drittens wird vorwiegend in narrativen Definitionsentfaltungen medialer Magazinberichte das spezifische Krankheitskonzept >Diagnose im institutionell verwendeten Krankheitsklassifikationssystem ‘ mit dem im Fallbeispiel geschilderten Symptomen verbunden, dadurch, dass die Person am Ende der Erzählung die Diagnose >Burnout` erhält und meist auf der Grundlage dieser Diagnose krankgeschrieben und in Behandlung geschickt wird. Dieses spezifische Krankheitskonzept wird dadurch in der Tat, wie im obigen SPIEGEL-Zitat angedeutet, hauptsächlich in fachexternen Texten mit >Burnout `verknüpft. In anderen fachexternen Medientexten wird eben diese Verbindung aber auch kritisiert und mit Bezug auf Fachleute negiert (z. B. SZ, 22.10.2011, = Weber 2011: 24, im QV unter 8.1.6).

803 Beispielbelege Unterstreichungen T.S.): „extrem stark ausgeprägtes Perfektionismusstreben“ (Wilms 2009, PiD); „Bei länger anhaltender Überforderung kann der Begriff Burnout [...] genutzt werden“ (Schramm/Berger 2013, Der Nervenarzt); langandauernde Stressreaktion (Schmitz 1998, PiEU); Belege im QV unter 8.1.4.

804 So impliziert das häufig zitierte Symptom Überforderung den antonymen Gegenpol der Unterforderung und beide Terme beziehen sich auf einen GEMEINSAMEN DURCHSCHNITTSWERT: ।weder zu hohe noch zu niedrige Anforderung an x/sich/etwas stellen (zur Antonymie vgl. Schippan 22002: 215); Adjektive wie dysfunktional, gefühllos oder unausgeglichen, die man als Zuschreibungen zum Burnout-Zustand findet, evozieren (gradierbar-)komplementäre Gegenterme (funktional, gefühlvoll, ausgeglichen) und eine Wertungspolarität, da die zuletzt genannten implizierten Gegenterme „das (völlige oder weitgehende) Fehlen der unerwünschten Eigenschaft“ markieren (Rachidi 1989: 93). Zu weiteren Beispielen vgl. Kap. 6.2.4.1.

805 Beispielbeleg: „Zu Beginn seiner Karriere war der Berliner Professor für seine Studenten jederzeit zu sprechen. [...] Heute beschränkt der inzwischen 53jährige Professor seine Begegnungen mit Studenten [...] auf ein kühles Minimum, [...].“ (DER SPIEGEL 1988: 162, Unterstreichungen T.S., im QV unter 8.1.6).

806 Vgl. zum Beispiel die semantischen Dimensionen „Agonalität der expliziten Gegenüberstellung“ - indiziert u. a. durch adversative und konzessive Konnektoren - oder „Agonalität der zeitlichen Gegenüberstellung“ - indiziert u. a. durch Temporaladverbien und Tempuswechsel (Mattfeldt 2018: 70ff. und 73ff.). 
- Doch auch fachliche Texte sind viertens nicht gänzlich unbeteiligt an dieser Zuordnung zum Bereich der Krankheitsdiagnostik, da sie >Burnout $<$ innerhalb ihrer fachkulturellen Praktiken und Textsorten beschreiben und dadurch gewissermaßen ,eingemeinden'. Dies sei an folgendem Beispiel verdeutlicht: Wenn der Titel einer Studie „Differentialdiagnose Burnout“ lautet, so könnte der Titel zunächst so interpretiert werden, dass hier beschrieben wird, wie man $\mathrm{zu}$ einer solchen Differentialdiagnose gelangt. Dass diese Studie aber zu dem Ergebnis kommt, dass die bisher „,verwendeten BurnoutMessinstrumente [...] nicht differential-diagnostisch validiert sind“ (vgl. den HTA-Bericht von Korczak/Kister/Huber 2010: 1, im QV unter 8.1.5) ${ }^{807}$, erfährt der Leser erst, wenn er diese Studie zu lesen beginnt. >Burnout ‘ wird in dieser Studie nach allen Regeln fachkultureller Vorgaben für Differentialdiagnosen überprüft und dadurch im Rahmen dieser Wissensstrukturierung perspektiviert und behandelt.

Vor dem Hintergrund der Ausführungen des letzten und dieses Kapitels bleibt schließlich zu fragen, welchen heuristischen Mehrwert dieses Modell für linguistische Diskursanalysen birgt. Dieser Frage wird im letzten Kapitel dieser Arbeit zusammen mit der Überprüfung der Ausgangshypothesen dieser Arbeit (Kap. 1.1 und 4.3.4) und der Reflexion über Anschlussstudien nachgegangen.

\subsection{Resümee und Ausblick}

In dieser Arbeit wurde am Beispiel des Burnout-Diskurses ein Modell einer diskursiven Praxis des Definierens aus intra- und transtextueller Perspektive entwickelt, um die Ausgangsfrage aus Kapitel $1.1 \mathrm{zu}$ bearbeiten, wie in Fach-, Medienund Vermittlungstexten der letzten vierzig Jahre ein spezifikationsbedürftiges Phänomen aus dem Bereich psychischer Gesundheit und Krankheit immer weiter eingegrenzt und damit definiert wird - einerseits punktuell in einzelnen fachlichen und fachexternen Erscheinungsformen des Diskurses und andererseits transtextuell über die Jahrzehnte hinweg. Durch die überindividuelle, transtex-

807 Zum Thema „Burnout“ sind zwei HTA-Berichte erschienen: Zur „Differentialdiagnostik des Burnout-Syndroms“ (Korczak/Kister/Huber 2010) und zur „Therapie des Burnout-Syndroms“ (Korczak/Wastian/Schneider 2012). Die Abkürzung HTA geht zurück auf „Health Technology Assessment“. Unter HTA wird nach der Bundesärztekammer die „systematische, evidenzbasierte Bewertung medizinischer Verfahren und Technologien im Hinblick auf deren Effekte auf die Gesundheitsversorgung verstanden.“ https://www.bundesaerztekammer.de/aerzte/qualitaetssicherung/health-technology-assessment/ (Zugegriffen: 30.9.2019). 
tuelle Betrachtungsweise des Definierens-Prozesses konnte transparent gemacht werden, wie eine Sachverhaltskonstitution in Form einer Definitionsvariante im Wechselspiel unifizierender und agonaler Praktiken beginnt, sich diskursiv durchzusetzen: erstens dadurch, dass der Diskurs um konkurrierende Sachverhaltskonstitutionen verknappt wird und zweitens, indem einzelne als solche bezeichnete „Kernmerkmale“ und wiederkehrende Referenzgrößen und Bezugsnormen im Kontext fachkultureller und medialer Praktiken über Einzeltexte hinweg wiederholt transportiert und damit verfestigt werden. Das Analysekonzept der >Unifizierung`, das dem Konzept der `Agonalität` in dieser Arbeit gegenübergestellt wurde, ermöglicht es, zwischen sprachlichen Mitteln und Praktiken explizit-intendierter Konsensfindung und sprachlichen Mitteln und Praktiken der unwillkürlichen, nicht explizit intendierten Verfestigung und Diskursverknappung zu unterscheiden. Aus dieser Analyseperspektive heraus werden zum einen sprachliche Mittel und Praktiken fokussiert, durch welche die Multiperspektivität bezogen auf ein Diskursthema reduziert wird, und zum anderen Spuren an der Textoberfläche gesichtet, die auf weitgehend unumstrittenes Orientierungswissen und gemeinhin akzeptierte Werte in einer Gesellschaft verweisen.

Der direkte Vergleich von Fachtexten verschiedener medizinischer und psychologischer Teilfächer und fachexternen Texten verschiedener Zeitungen, Zeitschriften und Onlineplattformen ergab weite Überschneidungen in den Erwartungen, die an den Akt des Definierens in Bezug auf ein psychisches Krankheitsbild von fachlicher und fachexterner Seite gestellt werden (Kap. 4.2.2). Das lässt sich darauf zurückführen, dass fachexterne Medientexte die Kritik an der Güte der Burnout-Definition insbesondere aus den klinischen Fächern (Psychiatrie, Medizin) zitieren und reformulieren und diese Ansprüche in den Bereich medialer Praxis übertragen, wie die Analyse sprachlicher Mittel des Definierens im fachlichen und fachexternen Diskursstrang gezeigt hat (Kap. 6.2.2 und 6.2.3).

Vornehmlich auf der Ebene der Morpheme und (Mehr-)Worteinheiten findet man Überschneidungen in den sprachlichen Mitteln der fachlichen und fachexternen Textsorten, die implizit eine Normalitätsfolie evozieren, vor deren Hintergrund die charakteristischen PHYSISCHEN UND PSYCHISCHEN SYMPTOME, VERHALTENSMERKMALE, EIGENSCHAFTEN und UMGEBUNGSFAKTOREN DES ARBEITSUnd PRIVATlebens von PERSONEn MIT BuRnout spezifiziert werden (siehe Kap. 6.2.2-6.2.3). Fachextern erfolgt die Abgrenzung allerdings sprachlich expliziter und variantenreicher: Vor allem in narrativen Formen des Definierens wird parallel auf verschiedenen sprachlichen Ebenen die Entwicklung oder der Kontrast zwischen Anfangs- und Endzustand entfaltet. Weitere verfestigte Positionen des Unifizierungsprozesses im Burnout-Diskurs sind z. B. die folgenden:

- >Burnout wird in den analysierten fachlichen und fachexternen Definitionstypen beinahe durchgängig auf die Arbeitswelt bezogen; in fachlichen Hand- 
und Lehrbüchern/Enzyklopädien und Fachzeitschriften erscheint es als ein Konzept, welches neben dem Arbeitsbezug zusammen mit den Themen „Belastung“, „Beanspruchung“, „Stress/Stressoren“, „(psychische) Gesundheit“, „Ressourcen(verlust) und Anforderungen“ verhandelt wird (siehe Kap. 6.2.4.1); es wird im fachinternen Diskursstrang vor allem den Fachbereichen an der Schnittstelle von (Arbeits-)Medizin und (Arbeits-)Psychologie/Soziologie und anwendungsbezogenen Fächern wie der Gesundheitswissenschaft oder pädagogischen Psychologie (bezogen auf Lehrergesundheit) zugerechnet.

- In den fachexternen Zeitungs- und Zeitschriftentexten erscheint >Burnout meist zusammen mit den Themen: „chronischer Stress/Disstress“, „,andauernde Belastungen“, „hohe/steigende Anforderungen (quantitativ und qualitativ) in der Arbeit/modernen Arbeitswelt oder in bestimmten Berufsfeldern“, „Überforderung“, „Nennung bestimmter persönlicher und arbeitsplatzbezogener Eigenschaften als Risikomerkmale“, „ansteigende Fehlzeiten/Arbeitsunfähigkeit aufgrund psychischer Krankheiten“, „prominente Persönlichkeiten mit Symptomen des Burnouts - besonders oft im Leistungssport“, „Stressbewältigung/Entspannung“ (siehe Kap. 6.2.3.1); bei den thematischen Ressorts (neben den übergreifenden Ressorts Nachrichten und Landkreise) stechen die Ressorts der Bereiche Wirtschaft, Bildung, Karriere und Sport heraus (siehe Kap. 6.2.4.1) .

- Auch wenn Variationen des Satzes Burnout kann jeden treffen den BurnoutDiskurs durchziehen, so werden dennoch sowohl im fachlichen als auch fachexternen Diskursstrang bestimmte Personengruppen als 'besonders betroffen bzw. gefährdet' sprachlich gekennzeichnet (z. B. durch Formulierungen wie Syndrom, von dem vor allem Angehörige sozialer Berufe betroffen sind oder wird oft von Menschen erlebt, die ... oder nicht nur Manager oder Leistungssportler, sondern auch ... etc.). Zu den besonders häufig beschriebenen und sprachlich hervorgehobenen Betroffenengruppen zählen demnach fachintern und -extern: Personen in sozialen/pflegenden/pädagogischen Berufen, Personen mit hoher Verantwortung/Motivation/Bereitschaft zur „Aufopferung“808/Leistungserwartung (z.B. im Leistungssport, mit Führungsverantwortung oder in der Politik) oder Personen mit hohem Ehrgeiz/ Perfektionismus oder mit hohen/idealistischen Erwartungen an den Beruf (siehe Kap. 6.2.4.1).

808 Die explizite Opfermetaphorik findet man weniger in fachlichen und stärker in fachexternen Medien- und Vermittlungstexten: „Hinze opferte mehr und mehr Freizeit“ (Falkai 2016: 20, G\&G, im QV unter 8.1.7); „Gefährdet sind auch Menschen in sozialen und pflegerischen Berufen, die sich für andere aufopfern“ (Kals 2004: 55, FAZ, im QV unter 8.1.6). 
- >Burnout sird über verschiedene Texte hinweg in exponierten Textpositionen als 'dreidimensionales' Konzept mit dem Hauptsymptom >Erschöpfung، konstituiert (dieses zeigt sich 'physisch' und 'psychisch' und in mehrfach 'gesteigerter Form' ('andauernd' und 'hohe Intensität')), mit ('gefühltem' und/oder 'tatsächlichem') >Leistungsverlust` und >Depersonalisation/Zynismus`. Das letzte Symptom wird als 'gesellschaftlich/beruflich inadäquat' konstituiert.

- Weitere zusammen mit den Hauptsymptomen häufig genannte Symptome sind sowohl fachintern als auch fachextern: Schlafstörungen, unspezifische somatische Beschwerden wie z. B. Kopf- und Rückenschmerzen, Magen- und Darmprobleme und Beschreibungen einer sgestörten Fähigkeit, sich von der Erschöpfung zu erholen`, negative Emotionen.

- >Burnout ‘ führt zu >(gefühlten) Einschränkungen oder zum Verlust in Bezug auf vorherige (in hohem Maße gezeigte) Fähigkeiten und Leistungen ‘ und

- >Burnout führt $\mathrm{zu}$ einer sTransformation von (in gesteigerter Form) gesellschaftlich positiv bewerteten Gefühlen/Eigenschaften/Einstellungen zu (in gesteigerter Form) negativen Gefühlen/Einstellungen der Arbeit und anderen Menschen gegenüber«.

- >Burnout wird in fachlichen und fachexternen Texten in Zusammenhang gebracht mit ${ }^{809}$ einem >Ungleichgewicht bzw. einer negativen Bilanz zwischen Anforderungen und Ressourcen` oder einem `Ungleichgewicht zwischen investierten Ressourcen und Ressourcengewinnen

Unterschiede zwischen verschiedenen fachlichen und fachexternen Formen des Definierens zeigen sich vor allem auf der Satz- und Textabschnittsebene der Definitionsentfaltung und einem damit zusammenhängenden Metakonzept (〉Gattung८, >Endzustand`, >Ereignis`, >Vorgang $)^{810}$ sowie auf der Ebene der definitorischen Grundfunktion (repräsentativ, repräsentativ-direktiv, deklarativ), einzelner Konzeptattribute, der Reflexion definitorischer Geltungsbedingungen

809 In fachexternen Kontexten wird häufig ein kausaler Zusammenhang konstituiert; in Fachtexten wird dieser Kausalzusammenhang entweder mit Vorbehalt präsentiert (z. B. indem noch weitere Forschung gefordert wird, vgl. Kissling/Mendel/Förstl 2014: 2588, DMW, im QV unter 8.1.4), oder es wird nur ein korrelativer Zusammenhang festgestellt (vgl. Reime/Steiner 2001: 304, PPmP, im QV unter 8.1.4).

810 Diese Metakonzepte weisen trotz ihrer Unterschiede jedoch auch Ähnlichkeiten auf: Denn das Metakonzept `Zustand in deskriptiven (stilistisch komprimiert verfassten) Definitionsentfaltungstypen entspricht zumeist einem `Endzustand ‘, der durch nominalisierte oder adjektivierte telische Vorgangs-/Tätigkeitsverben charakterisiert wird (Erschöpfung $(<$ (sich/etw.) erschöpfen/erschöpft werden), Depersonalisation (< depersonalisieren), reduzierte Leistungsfähigkeit ( $<$ Leistungsfähigkeiten werden reduziert/reduzieren sich), Überlastung ( $<$ (sich) überlasten)). 
(Kap. 6.2.2.4, 6.2.3.3 und 6.2.4.2) und der diskursiven Wirkmacht einzelner Definitionsvarianten (siehe Kap. 7.2)..$^{811}$

Durch das vorgestellte Analysemodell, das in eine Typologie diskursiver Praxis des Definierens mündet, konnten die Unterschiede zwischen den definitorischen Entfaltungstypen und Grundfunktionen, aber auch deren Verschränkungen sichtbar gemacht werden (Kap. 7.2). Diese Ergebnisse veranschaulichen, dass Definitionen im Burnout-Diskurs im Spannungsfeld von multiperspektivischer, falsifikatorischer Wissensaushandlung und notwendiger Einigung für die Praxis stehen: auf der einen Seite findet man unterschiedliche Sachverhalts- und Bedeutungsfixierungsversuche (von denen sich manche aufgrund ihrer klassischen, komprimierten Form und durch Gewichtungs- und Verknappungspraktiken eher diskursiv durchsetzen als andere) und auf der anderen Seite steht das regulative Ideal der Sachverhalts- und Bedeutungsfixierung. In Wissensbereichen, in denen verbindliche Definitionen für die institutionell eingebundene Praxis unabdingbar sind, wie im Bereich (psychischer) Gesundheit und Krankheit, kann das regulative Ideal so stark sein, dass der Anspruch der 'Allgemeingültigkeit' auch auf Definitionsformen mit repräsentativem Geltungsanspruch übertragen wird.

Wie durch die Analysen in Kapitel 7.3. dargelegt wurde, setzen semantische Kämpfe gerade an diesen Übertragungspunkten definitorischer Geltungsansprüche im Burnout-Diskurs an. So sind Definiens-Nomina (Syndrom, Krankheit, Diagnose), die >BURNOUT، aus dem falsifikatorischen Spiel der Wissensaushandlung in den Kontext deklarativer gesellschaftlicher Einigung und darauf basierender Kostenübernahme überführen, im Burnout-Diskurs besonders umkämpft. Insofern lässt sich durch die wechselseitige Analyse unifizierender und agonaler Dimensionen herausarbeiten, welche Teilbedeutungen der im Diskurs essenziellen Grundkonzepte >Gesundheit` und >Krankheit` umkämpft sind und welche nicht.

Krankheits- und Gesundheits-Attribute wie die folgenden werden sowohl in vielen fachinternen als auch fachexternen Texten versprachlicht (siehe Kap. 6.2.4.2): z. B. für das Konzept `Krankheit` die Attribute 'subjektives Leiden', 'Symptome als realsemantische (Warn)signale' oder '(drohende) eigendynamisch sich entwickelnde Funktions- und Handlungseinschränkungen' (vgl. Kap. 3.2 und 6.2.4.2); für das Konzept >Gesundheit ‘ lassen sich die im Folgenden nach Faltermaier zitierten Konzeptualisierungen von >Gesundheit‘ sowohl in fachlichen als auch fachexternen Texten des Burnout-Diskurses nachweisen:

811 Die Wirkmacht einzelner Definitionen kann nur aus chronologischer, transtextueller Perspektive nachvollzogen werden. Die einzelnen Schritte dieser Analyse sind im Modell einer diskursiven Praxis des Definierens in den Fragen 7-11 dokumentiert (siehe Kap. 7.2). 
Gesundheit als psychisches Wohlbefinden: Sie wird beschrieben als innere Ruhe und Ausgeglichenheit sowie als positive Stimmung, Lebensfreude und Zufriedenheit, gelegentlich auch im umfassenden Sinn als inneres Gleichgewicht.

Gesundheit als körperliche und/oder geistige Leistungsfähigkeit im Hinblick auf die gute (z. B. optimale) Erfüllung von zentralen Aufgaben und sozialen Rollen (z. B. in der beruflichen Arbeit, im Sport) sowie als Handlungsfähigkeit im Hinblick auf grundlegende Funktionen des alltäglichen Lebens (z. B. am Morgen gut aufstehen können; normal essen, trinken, sich bewegen und schlafen können).

(Faltermaier 2005: 37, Kursivsetzung im Orig., vgl. Kap. 3.2)

Nicht durchgängig realisiert, wie auch umstritten sind hingegen die KrankheitsAttribute der 'Hilfs- und Behandlungsbedürftigkeit' und 'Arbeitsunfähigkeit'. Gegen diese Attribute wird mit Benennungssubstitutionen (Risikozustand, Ausweich-/Zusatzdiagnose, Erschöpfungs- und Überforderungssyndrom statt Krankheit, Diagnose) und Überschriften wie Modediagnose Burnout oder Useful illness (vgl. Kap. 1.2) angeschrieben. Hieran wird deutlich, dass gegenwärtig am Gegenstand BuRNout sozialversicherungsrechtlich ausgehandelt wird, wo die Grenze der 'Behandlungsbedürftigkeit' und 'Arbeitsunfähigkeit' für Erschöpfungs-Leiden und psychosomatische Beschwerden verläuft, für die keine eindeutigen organischen Ursachen gefunden werden können. Damit hängt zudem die Frage zusammen, welche gesellschaftlichen Instanzen für die Aufrechterhaltung oder „Wiederherstellung der Erwerbsfähigkeit“ (Geisthövel 2019: 57) zuständig sein sollen. Diese Diskussionen beginnen spätestens 1884 mit Einführung der gesetzlichen Unfallversicherung und werden bis weit hinein ins 20. Jahrhundert unter verschiedenen Bezeichnungen geführt: wie z. B. Rentenneurose, Organneurose, vegetative Dystonie (ebd.: 49; 58), Neurasthenie als „nervöse Veranlagung“ bzw. „endogene Reaktionsform“ oder als „exogene Reaktionsform“ einer „neurasthenischen Reaktion des vorher Gesunden“ bzw. „Tätigkeitsneurose“ (z. B. Sterz 1928: 19-20, Kursivsetzung im Orig., im QV unter 8.1.5).

In Kapitel 6.2 dieser Arbeit wurde dargelegt, dass die fach- und allgemeinsprachliche Verwendung des entlehnten Substantivs Burnout in einer Zeit in Deutschland aufkommt, in der die „Loslösung“812 von Termini rund um die Neurosenlehre und von psychoanalytisch-ätiologischen Modellen der „Konfliktsu-

812 Schuster beschreibt im Rahmen der Wortschatzentwicklung der psychiatrischen Schreibpraxis im 19. Jahrhundert, dass einige „Formen lexikalischen Wandels“ und „die Verwendung neuer Lexeme und die Bevorzugung bestimmter Lexeme auch dadurch bedingt ist, dass Loslösung - verstanden als Loslösung vom Diachronie- und Rahmenbezug - [...] signalisiert wird“ (Schuster 2010: 286). 
che“ (Geisthövel 2019: 58) schon begonnen hat. Mit dem Erscheinen der „zweite[n] Überarbeitung des Diagnostic and Statistical Manual of Mental Disorders“ im Jahr 1980 wurde des Weiteren der „Wechsel von ätiologisch-theoretischen zu deskriptiv-diagnostischen Klassifikationskriterien“ vollzogen ${ }^{813}$ (ebd.: 59). Der neue Begriff `Burnout wird selbst von Freudenberger, der diskursiv als Entdeckerfigur konstituiert wird, jenseits der damals noch üblichen Klassifizierungsvokabeln psychotisch und neurotisch platziert, ${ }^{814}$ und der Diskurs speist sich lexikalisch aus neuen Fach- und Forschungsrichtungen. ${ }^{815}$

Doch die alten Streitpunkte nach den Ursachen für die Erschöpfungszustände und die damit verbundenen Verantwortlichkeitskämpfe kehren zurück: So lässt sich die folgende aktuell von psychiatrischer Seite vorgetragene Kritik mit der oben aus den 1920er Jahren zitierten Unterscheidung einer exogenen und endogenen neurasthenischen Reaktion vergleichen: Durch die „Berichterstattung in den Medien“ über >Burnout werde eine „Krankheitsdefinition gefördert“, die >Burnout` mit ,einer Erkrankung der Leistungsträger und der ,Starken“ gleichsetzt“ (DGPPN 2012: 1), die „,vorher für etwas gebrannt haben“ und nun ein „Verwundetenabzeichen“ tragen (Kämmerer 2016: 87, PiD, im QV unter 8.1.4), den Begriff >Depression` dagegen mit ,einer Erkrankung der (anlagebedingt) ,Schwachen“ verknüpft“ (DGPPN 2012: 1, im QV unter 8.1.9). Der in dieser Arbeit aus diachroner und transtextueller Perspektive beschriebene Definierensprozess hat gezeigt, dass der angenommene (kausale) Zusammenhang zwischen >Arbeitsüberlastung` oder seiner negativen Ressourcen- bzw. Erwartungsbilanz

$813 \mathrm{Saß/Saß-Houben} \mathrm{führen} \mathrm{zur} \mathrm{Entwicklung} \mathrm{der} \mathrm{klinisch-psychologischen} \mathrm{und} \mathrm{psychiatri-}$ schen Klassifikation in der ICD und dem DSM aus, dass es eine „pragmatische Konsequenz“ war, „ätiologieneutrale, rein deskriptive und anhand von Kriterien operationalisierbare Diagnosesysteme zu schaffen, in denen bestimmte Symptomcluster per definitionem zu Störungsbildern zusammengefasst werden“ (Saß/Saß-Houben 2005: 145, vgl. Kap. 3.2).

$814 \mathrm{Zu}$ Beginn des Buchs „Burn-out - The High Cost of High Achievement“ schreiben Freudenberger/Richelson (1980a: xv, im QV unter 8.1.5): „Many of the people who come to my office describe similar feelings, and yet they are neither neurotic nor psychotic in the historical sense of these words. That they are suffering is undeniable“ (Unterstreichungen T.S.).

815 Zentral sind beispielsweise Arbeitsanalysen und die Forschungsarbeiten zu 'psychischer' >Belastung` und `Beanspruchung`, `Berufsstress`, `Arbeitszufriedenheit` und `Arbeitsmotivation im Umfeld der Arbeits- und Organisationspsychologie, die sich historisch u.a. aus der Human-Relations-Bewegung und dem Human-Resource-Management entwickelt hat (siehe Kap. 5.2). Große Bedeutung haben des Weiteren medizinsoziologische und arbeitspsychologische Modelle wie die „Gratifikationskrise“ oder das „Job-Demands-Resources-Modell“ (vgl. Kap. 6.2.4.1 und 6.2.4.2). Die im Kontext dieser Modelle häufig verwendeten Vokabeln Belastung, Beanspruchung, Stress, Überlastung und Ressourcen werden sowohl in fachlichen Texten als Termini (teilweise mit Rückbindung an DIN-Normen) als auch in fachexternen Texten im Rahmen alltagssemantischer Zusammenhänge gebraucht (siehe Kap. 5.2, 5.3 und 7.2). 
im Arbeitskontext $\triangleleft$ und psychosomatischen Symptomen und Verhaltensmerkmalen in den meisten Definierensformen präsent ist. >Burnout ‘ wurde bei der Zusammenführung der drei im Diskurs am häufigsten genannten Kernsymptome mit der ICD-10 (siehe Abb. 20 in Kap. 6.2.4.2) bzw. der ab 2022 geltenden ICD-11 (siehe Beleg 12 in Kap. 6.2.2.1) zwar in den Anhang des Klassifikationssystem verlagert, das Konzept nimmt jedoch von dort einen recht stabilen Platz als 'ätiologische Zusatzdiagnose' ein, deren Anwendung Praktikerinnen und Praktikern seit einigen Jahren von Fachgesellschaften wie der DGPPN trotz mancher Kritikpunkte sogar empfohlen wird:

Die DGPPN empfiehlt deshalb, immer zuerst die jeweils zutreffende ICD-10-Diagnose (z. B. Depression oder Hypertonie) zu verschlüsseln. Wenn darüber hinaus angenommen wird, dass bei der Entstehung und Aufrechterhaltung dieser als Hauptdiagnose festgestellten Erkrankungen ein Erschöpfungszustand im Sinne eines Burnouts eine entscheidende Rolle gespielt hat, sollte zusätzlich die ICD-10-Anhangsziffer Z 73.0 codiert werden. Dadurch werde die krankheitsauslösende Überforderung eines Patienten gekennzeichnet und entsprechende spezifische Therapiemaßnahmen nahegelegt. ${ }^{816}$

Kissling/Mendel/Förstl (2014: 2588, DMW, im QV unter 8.1.4, Unterstreichungen T.S.)

Ist Burnout vor dem Hintergrund der historischen Ausführungen also doch, wie im Diskurs kritisch angemerkt wird, ,old wine in a new bottle“ (Maslach 1982: 29) oder „allenfalls ein anderes Wort für Depression“"818?

Die Diskussionspunkte, die früher im Kontext von Neurasthenie, Rentenoder Tätigkeitsneurose verhandelt wurden und sich heute im Kontext des Burnout-Diskurses wiederfinden, sowie die Kritik an der fehlenden oder zu undifferenzierten Abgrenzung vom Diagnosekonzept der >Depression ${ }^{819}$ legen diesen

816 Ähnliche Empfehlungen gibt die DGPPN in ihrem Positionspapier zu „Burnout“ aus dem Jahr 2012 (= DGPPN 2012: 3ff., im QV unter 8.1.9); vgl. auch Weimer/Kraus (2011: 242, Der Psychotherapeut, im QV unter 8.1.4); Breitenbürger (2012: 238, Dt. Ärzteblatt, im QV unter 8.1.4); Berger/Falkai/Maier (2012: 213, Dt. Ärzteblatt, im QV unter 8.1.4); Riedel-Heller/Luppa/Seidler et al. (2013: 834, Der Nervenarzt, im QV unter 8.1.4); Nach Kaschka/Korczak/Broich hingegen sollte „Burn-out“ „möglichst nicht als Diagnose und Grundlage für sozialrechtliche Entscheidungen herangezogen werden. (Kaschka/Korczak/Broich 2011: 781, im Dt. Ärzteblatt, im QV unter 8.1.4). 817 Maslach 1982, im QV unter 8.1.5; vgl. zu diesem Topos `Altes in neuem Gewand 1984: 211/212 im QV unter 8.1.5 und den Abschnitt „B) Anspruch auf Trennschärfe und Deutlichkeit“" in Kap. 4.2.2).

818 Blech 2012, im SPIEGEL 6/2012: 142, im QV unter 8.1.6.

819 Vgl. folgendes Zitat in der ZEIT, warum viele Fachleute zunächst keine Notwendigkeit sahen, `Burnout ‘ zu definieren: „Viele Ärzte und Psychotherapeuten waren zwar der festen Überzeugung, Burn-out sei nur ein neuer Name für Depressionen, benutzten das Wort aber dennoch gern.“ (DIE ZEIT, 01.12.2011, = Albrecht 2011a, im QV unter 8.1.6, Unterstreichung T.S.). Es gibt aber auch fachliche Stimmen, die Unterschiede zwischen `Burnout $\triangleleft$ und `Depression anführen (siehe dazu Kap. 6.2.2.2, 6.2.4.2 und 6.2.3.1). 
Schluss nahe. So müsste man auch dem Psychiater Falkai zustimmen, der einige Symptom-Benennungen aus dem Diagnosekatalog für `Depression` mit den sich durchgesetzten Symptombenennungen des Konzepts >Burnout in folgendem Zitat gleichsetzt:

\begin{abstract}
Betrachtet man den Symptomkatalog [der Diagnose Depression, Erg. T.S.], so trifft einiges auch auf das Burnout-Syndrom zu. Dessen zentrales Merkmal, »anhaltende Erschöpfung und Leistungsminderung", meint letztlich nichts anderes als das Hauptsymptom »Antriebsmangel und erhöhte Ermüdbarkeit« im Diagnosekatalog für Depressionen.
\end{abstract}

(Falkai 2016: 24, G\&G, im QV unter 8.1.7, Unterstreichung T.S.)

Über die inhaltliche Ähnlichkeit der Symptomatik aus klinisch-psychiatrischer Sicht kann und soll hier keine Aussage getroffen werden. Aus linguistischer Sicht und mit Blick auf die starken Definitions-Bemühungen, die sich im BurnoutDiskurs manifestieren, scheint es jedoch ein (bekanntes, wenn auch vermeintlich überwundenes) gesellschaftliches und teilweise fachliches Bedürfnis nach (ätiologischer) Differenzierung zwischen Ermüdungs- bzw. Erschöpfungszuständen zu geben, und diese verschiedenen Perspektivierungen manifestieren sich an der Sprachoberfläche. Bei der obigen Gegenüberstellung im Zitat von Falkai ist nicht eindeutig zu erkennen, welche syntaktischen Einheiten der Autor direkt aufeinander bezieht. Vergleicht man zum Beispiel die Formulierungen erhöhte Ermüdbarkeit und anhaltende Erschöpfung im Hinblick darauf, welche semantischen Aspekte durch die Wahl der sprachlichen Zeichen bei den Symptomen jeweils besonders akzentuiert werden, so fällt Folgendes auf: Bei erhöhte Ermüdbarkeit ist die Basis des Substantivs ein Adjektiv auf -bar. ${ }^{820}$ Dadurch steht die personelle Eigenschaft oder Zustandsbeschreibung 'kann ermüdet werden' perspektivisch im Mittelpunkt, und die „faktische Möglichkeit“ (vgl. Blühdorn 2012: 50), dass diese Person ermüdet wird, ist 'erhöht'. Bei anhaltende Erschöpfung hingegen wird der Fokus auf den Endzustand eines telischen Prozesses/einer telischen Tätigkeit gelegt, in dem 'etwas (z. B. die Kräfte) erschöpft wurden/jmd. sich oder seine Kräfte erschöpft hat'. Überspitzt könnte man sagen: Die ausgebrannte Person ist in dieser Gegenüberstellung 'faktisch erschöpft bzw. ohne Kraft' und dieser Zustand 'hält an'. Bei der depressiven Person ist im Vergleich dazu bereits

820 Mit -bar können Adjektive aus Verben abgeleitet werden (Blühdorn 2012: 49). Das Suffix wird in der Duden-Grammatik den „passivisch-modalen Bildungstypen“ zugewiesen (DUDEN 72006: 765). Adjektive auf -bar geben demnach an, „was mit einer Sache getan werden kann“ (Blühdorn 2012: 49). Blühdorn erläutert zudem die Unterscheidung „zwischen faktischer Möglichkeit, Denkmöglichkeit und ethischer Möglichkeit“ als mögliche Lesarten des modalen Sinns (ebd.: 50). Im Kontext des obigen Beispiels (erhöhte Ermüdbarkeit) kommt nur die Lesart der faktischen Möglichkeit in Frage. 
die 'faktische Möglichkeit, ermüdet zu werden bzw. ohne Kraft zu sein', 'erhöht'. Auf Duden.de drückt sich dieser semantische Unterschied durch folgende Bedeutungsangaben aus: Für das Lemma Ermüdung findet man die Bedeutungsangabe „Neigung zu schneller Ermüdung“ und für das Lemma Erschöpfung die Angabe „durch übermäßige Anstrengung hervorgerufene Ermüdung“. ${ }^{821}$ Diese unterschiedlichen sprachlichen Perspektivierungen sind vor dem Hintergrund interessant, dass die Ausdrücke Ermüdbarkeit, ermüdbar im Untersuchungskorpus nur selten im Kotext zu Burnout auftreten. Wenn die Ausdrücke gemeinsam auftreten, dann wird zumeist im direkten Kotext auch ein Vergleich zur Depression wie im obigen Beispiel angestellt. ${ }^{822}$

Dieses Beispiel sollte zeigen, dass es sehr aufschlussreich sein kann, rekursive Symptom-Formulierungen bei semantisch ähnlichen Begriffen wie >Burnout und >Depression ‘n einer Anschlussstudie miteinander zu vergleichen. Dafür würde sich auch eine quantitativ unterstützte vergleichende korpuslinguistische Analyse signifikanter Lexeme in den Kotexten der in dieser Arbeit herausgearbeiteten sprachlichen Mittel der Graduierung, Quantifizierung und Polarisierung anbieten. Denn diese bewirken auf der Ebene der Morpheme und (Mehr)-Worteinheiten eine Abgrenzung zu normalen alltäglichen Erschöpfungszuständen bzw. zu normalem oder erwünschtem Verhalten und Erleben (vgl. Kap. 6.2.2.2 und 6.2.3.1). Der Vergleich könnte sichtbar machen, zu welchen biologischen und sozial-kulturellen Normen oder Werten `Burnout und `Depression` jeweils in Beziehung gesetzt werden. Dass `Burnout nicht nur auf ätiologischer, sondern auch auf symptomatischer Ebene eng mit kulturellen Normvorstellungen verbunden ist, zeigen beispielsweise Fragebogen-Items wie das folgende: Die Verneinung der Frage „Haben Sie in der Freizeit genug Energie für Ihre Familie und Freunde?“ (vgl. z. B. Kristensen/Borritz/Villadsen et al. 2005: 200, im QV unter 8.1.5) wird als Symptom gewertet. Auch in Medientexten wird häufig angeführt, dass die Beziehung zur Familie/ zu den Kindern „leidet“ (vgl. z. B. Abé 2012, SPIEGEL ONLINE, im QV unter 8.1.6). Diese Beispiele zeigen, welches Verhalten in einem Kulturkreis als 'normal' klassifiziert wird und wie eine Gesellschaft sich durch die Bemühungen, ein krankheits-

821 Siehe Ermüdbarkeit unter https://www.duden.de/rechtschreibung/Ermuedbarkeit und Erschöpfung unter https://www.duden.de/rechtschreibung/Erschoepfung (zuletzt eingesehen am 2.1.2020).

822 Siehe dazu den folgenden Beleg aus der Zeitschrift „Psychotherapie im Dialog“: „Während beim BOS Symptome wie depressive Verstimmung, Verlust von Freude und Interessen sowie rasche Ermüdbarkeit und reduzierter Antrieb durchaus vorkommen können, finden sich Schuldgefühle, reduziertes Selbstwertgefühl, Appetit- und Libidoverlust eher nicht. Je ausgeprägter ein BOS ist, umso höher ist die Korrelation mit einer depressiven Episode“ " (Beschoner/SchönfeldtLecuona/Braun et al. 2009, PID, im QV unter 8.1.4, Unterstreichungen T.S.). 
wertiges Konzept zu definieren, über ihren gemeinsamen Werte- und Normenkanon verständigt. Berufliche Rollenerwartungen kommen im Adjektiv herzlos zum Ausdruck, das im Burnoutdiskurs häufig verwendet wird, um das Merkmal 'Zynismus' im Kontext helfender Berufe zu umschreiben: Wenn über eine Pflegeperson im Rahmen einer Symptombeschreibung gesagt wird, sie handle herzlos, dann schwingt in dieser Aussage eine „generische Prädikation“ (vgl. Schmidt-Brücken 2015: 38ff.) bzw. sozial-kulturelle Praktik mit, die unter 'normalen' Umständen für diesen Berufskontext 'teilnehmende Fürsorge' vorsieht. ${ }^{823}$ So könnte beispielsweise ein kontrastiver quantitativ-hermeneutischer Vergleich von gradierbarkomplementären Adjektivpaaren ${ }^{824}$ (wie z. B. realistisch - unrealistisch; effektiv - ineffektiv) oder von Wortbildungsmustern mit dem Suffix -los (z. B. freudlos vs. lustlos, antriebslos vs. energielos) im Kotext von >Depression ‘ und >Burnout schlussreich sein. Interessant wäre vor diesem Hintergrund ferner eine vergleichende Ko(n)text-Analyse zu Sätzen oder Formulierungen wie Irgendwann ging gar nichts mehr/die Aufgaben nicht mehr bewältigen können/nicht mehr mit Freude an die Arbeit. Denn im Burnout-Diskurs werden durch (gradierbar-komplementäre) Gegensätze und Zustandsbeschreibungen im Kotext der Formel nicht(s) mehr kulturell-soziale und berufliche Ideal- und Tauglichkeitsnormen und Produktivitätsgrenzen verhandelt. ${ }^{825}$ Schließlich würde sich auch ein Vergleich anbieten, welche Personengruppen in fachlichen Texten im Kontext von >Burnout Kontext von >Depression « besonders erforscht werden und welche Personengruppen medial jeweils als besonders ‘betroffen' oder 'gefährdet' dargestellt werden.

Ergebnisse der vorliegenden Studie und die genannten diskurslinguistischen Anschlussstudien können auf diese Weise die kulturellen und sozialen Normen und Werte, die in der Einleitung zum Klassifizierungsmanual DSM-V als enge Bezugspunkte für die Definition psychischer Störungen benannt werden (Wittchen/Falkai/Stangier et al. ${ }^{2} 2018$ : 19, siehe auch Kap. 1.1), von sprachlicher Seite aus transparent machen. Es kann des Weiteren anhand grammatischsprachlicher Kategorien veranschaulicht werden, welche Sprachgebrauchsformen (in Gegenüberstellung $\mathrm{zu}$ anderen Formen) im Bereich psychischer

823 Vgl. dazu Köller (2004): „Bei der Verwendung des Negationssuffixes -los gilt in der Regel die Annahme, dass die jeweilige Eigenschaft nicht erwünscht ist“ (Köller 2004: 365f.).

824 Für das Konzept `Burnout` wurden diese in Kapitel 6.2.4.1 herausgearbeitet.

825 Siehe z.B., Unterstreichungen T.S.: „Flexibilität heißt das große Stichwort, und für den Beschäftigten heißt das, seine Freizeit flexibel für die Arbeit zu verplanen. Diagnose Burn-out. Immer mehr Menschen können diese Art von Flexibilität nicht mehr ertragen.“ (SZ, Bohsem 2012a: 17, im QV unter 8.1.6); „140 Überstunden im Monat. Wenn ich nach Haus kam, konnte ich nicht mehr abschalten“ (SPIEGEL ONLINE, 08.08.2012, Abé 2012). 
Gesundheit und Krankheit Stigmatisierungspotenzial bergen und in welchem Maß einzelne sprachliche Benennungsalternativen in Fragebogendesigns auf Wirk- und Störfaktoren hinweisen können. Eine vertiefende Zusammenarbeit zwischen psychiatrisch-diagnostischer Theorie und Praxis sowie zwischen empirischen Methoden der (klinisch)-psychologischen Testentwicklung und diskursund korpuslinguistischen Analyseverfahren wäre vor diesem Hintergrund für alle beteiligten Fächer erkenntnisstiftend und nutzbringend.

Die vorliegende Arbeit hat das Ziel verfolgt, die definitorischen Funktionsansprüche einer Diskursgemeinschaft und dominante Typen des Definierens für den Bereich psychischer Gesundheit und Krankheit am Beispiel des Phänomens BURNouT im Analysemodell einer diskursiven Praxis des Definierens zu erarbeiten. Diskurslinguistische Vergleichsstudien zu anderen Konzepten psychischer Gesundheit und Krankheit oder zu sachlich weiter entfernten Wissensbereichen könnten Aufschluss darüber geben, welche sprachlichen Mittel und Praktiken nur diskursgebunden und welche diskursübergreifend definitorische Funktionen erfüllen und von welchem weitgehend unumstrittenen, impliziten Orientierungswissen, je nach Diskurs, eine unifizierend-definitorische Wirkung ausgeht.

Den sprachlich-diskursiven Spuren eines solchen Praxis-Wissens im Bereich psychischer Gesundheit und Krankheit, das sich teilweise ,zwischen den Zeilen verbirgt', aber u.a. von dort seine definitorische Wirkmacht entfaltet, wurde in dieser Arbeit am Beispiel des >BURNOUT-SYNDROMS` auf den Grund gegangen. 